\title{
SPECTRA OF ORBIFOLDS WITH CYCLIC FUNDAMENTAL GROUPS
}

\author{
EMILIO A. LAURET
}

\begin{abstract}
We give a simple geometric characterization of isospectral orbifolds covered by spheres, complex projective spaces and the quaternion projective line having cyclic fundamental group. The differential operators considered are Laplace-Beltrami operators twisted by characters of the corresponding fundamental group. To prove the characterization, we first give an explicit description of their spectra by using generating functions. We also include many isospectral examples.
\end{abstract}

\section{INTRODUCTION}

Let $M$ be a connected compact Riemannian manifold, and let $\Gamma_{1}$ and $\Gamma_{2}$ be cyclic subgroups of Iso $(M)$. In this paper we will consider the question:

Under what conditions the (good) orbifolds $\Gamma_{1} \backslash M$ and $\Gamma_{2} \backslash M$ are isospectral?

Here, isospectral means that the Laplace-Beltrami operators on $\Gamma_{1} \backslash M$ and on $\Gamma_{2} \backslash M$ have the same spectrum. Such operators are given by the Laplace-Beltrami operator on $M$ acting on $\Gamma_{j}$-invariant smooth functions on $M$.

Clearly, the condition on $\Gamma_{1}$ and $\Gamma_{2}$ of being conjugate in $\operatorname{Iso}(M)$ is sufficient since in this case $\Gamma_{1} \backslash M$ and $\Gamma_{2} \backslash M$ are isometric. However, it is well known that this condition is not necessary due to examples of non-isometric isospectral lens spaces constructed by Ikeda [1k80a. Lens spaces are spherical space forms with cyclic fundamental groups, that is, they are the only compact manifolds with constant sectional curvature and cyclic fundamental group.

In the recent paper [LMR15], R. Miatello, J.P. Rossetti and the author show an isospectral characterization among lens spaces in terms of isospectrality of their associated congruence lattices with respect to the one-norm $\|\cdot\|_{1}$. More precisely, each cyclic subgroup $\Gamma$ of $G=$ $\mathrm{SO}(2 n)=\mathrm{IsO}^{+}\left(S^{2 n-1}\right)$ has associated a sublattice $\mathcal{L}_{\Gamma}$ of the weight lattice of $G$. Then, it is shown that $\Gamma_{1} \backslash S^{2 n-1}$ and $\Gamma_{2} \backslash S^{2 n-1}$ are isospectral if and only if for each $k \geq 0$ there are the same number of elements in $\mathcal{L}_{\Gamma_{1}}$ and $\mathcal{L}_{\Gamma_{2}}$ having one-norm equal to $k$.

The aim of this paper is to extend the results in [LMR15] to more general locally homogeneous compact Riemannian manifolds having cyclic fundamental group. Indeed, we will focus here on spaces covered by compact symmetric spaces of real rank one.

1.1. Method. We fix an arbitrary compact connected Riemannian manifold $(M, g)$. The spectrum of the Laplace-Beltrami operator $\Delta$ acting on smooth functions on $M$ is given by a sequence of eigenvalues

$$
0=\lambda_{0}<\lambda_{1}<\lambda_{2}<\ldots
$$

each of them with finite multiplicity

$$
\operatorname{mult}_{\lambda_{k}}(\Delta):=\operatorname{dim}_{\operatorname{Eig}_{\lambda_{k}}(\Delta)}
$$

Date: July 24, 2018.

2010 Mathematics Subject Classification. Primary 58J50, Secondary 58J53, $17 \mathrm{~B} 10$.

Key words and phrases. spectrum, isospectral, one-norm, lens space, cyclic fundamental group. 
Here, $\operatorname{Eig}_{\lambda_{k}}(\Delta)$ denotes the eigenspace of $\Delta$ with eigenvalue $\lambda_{k}$. The spectral zeta function of $\Delta$, also called Minakshisundaram-Pleijel zeta function, is given by

$$
\zeta_{\Delta}(s)=\sum_{k \geq 1} \operatorname{mult}_{\lambda_{k}}(\Delta) \lambda_{k}^{-s}
$$

Let $\Gamma$ be a discrete cocompact subgroup of $\operatorname{Iso}(M)$. The space $\Gamma \backslash M$ is compact and inherits a structure of good Riemannian orbifold $(\Gamma \backslash M, g)$, which is a manifold if and only if $\Gamma$ acts freely on $M$. The operator $\Delta$ commutes with isometries, thus the Laplace-Beltrami operator $\Delta_{\Gamma}$ on $(\Gamma \backslash M, g)$ coincides with $\Delta$ restricted to $\Gamma$-invariant smooth functions on $M$. Consequently, any eigenvalue in the spectrum of $\Delta_{\Gamma}$ is in the set $\left\{\lambda_{k}: k \geq 0\right\}$, and

$$
\operatorname{mult}_{\lambda_{k}}\left(\Delta_{\Gamma}\right)=\operatorname{dim} \operatorname{Eig}_{\lambda_{k}}(\Delta)^{\Gamma}
$$

Hence, $0 \leq \operatorname{mult}_{\lambda_{k}}\left(\Delta_{\Gamma}\right) \leq$ mult $_{\lambda_{k}}(\Delta)$ and $\lambda_{k}$ is an eigenvalue of $\Delta_{\Gamma}$ unless $\operatorname{dim} \operatorname{Eig}_{\lambda_{k}}(\Delta)^{\Gamma}=0$.

Following an idea of Ikeda, we encode the spectrum of $\Delta_{\Gamma}$ via the following formal power series

$$
F_{\Delta_{\Gamma}}(z):=\sum_{k \geq 0} \operatorname{mult}_{\lambda_{k}}\left(\Delta_{\Gamma}\right) z^{k}
$$

that we called the spectral generating function associated to $\Gamma \backslash M$. Clearly, $\Gamma_{1} \backslash M$ and $\Gamma_{2} \backslash M$ are isospectral if and only if $F_{\Delta_{\Gamma_{1}}}(z)=F_{\Delta_{\Gamma_{2}}}(z)$.

We now assume that $M=G / K$ is a compact homogeneous Riemannian manifold and $\Gamma$ is a cyclic subgroup of $G$ contained in a (fixed) maximal torus $T$. These hypotheses make easier the explicit computation of $\operatorname{dim} \operatorname{Eig}_{\lambda_{k}}(\Delta)^{\Gamma}$. Indeed, $\operatorname{Eig}_{\lambda_{k}}(\Delta)$ is a finite dimensional representation of $G$, so it decomposes diagonally into weight spaces with respect to $T$. Hence, $\operatorname{Eig}_{\lambda_{k}}(\Delta)$ is the sum over the set $\mathcal{L}_{\Gamma}$ of $\Gamma$-invariant weights of the corresponding weight spaces. Consequently, its dimension is equal to the sum over elements in $\mathcal{L}_{\Gamma}$ of the corresponding multiplicities. In conclusion, the determination of mult $_{\lambda_{k}}\left(\Delta_{\Gamma}\right)$ reduces to computing the multiplicities of the elements in $\mathcal{L}_{\Gamma}$ in the representation $\operatorname{Eig}_{\lambda_{k}}(\Delta)$ of the group $G$.

1.2. Results. For compact symmetric spaces of real rank one, the representations $\operatorname{Eig}_{\lambda_{k}}(\Delta)$ for $k \geq 0$ are spherical representations, which are well known. Their multiplicities satisfy a simple (geometric) formula when $M=P^{n}(\mathbb{C}), S^{2 n}, P^{1}(\mathbb{H})$ or $S^{2 n-1}$ for $n \geq 1$ (Proposition 3.2). More precisely, in the weight lattice associated to $G=\mathrm{SU}(n+1), \mathrm{SO}(2 n+1), \mathrm{Sp}(2)$ or $\mathrm{SO}(2 n)$, there is a norm $\|\cdot\|$ such that the multiplicity of a weight depends only on its norm value. Such norm is the maximum norm $\|\cdot\|_{\infty}$ for $\operatorname{Sp}(2)$ and the one-norm $\|\cdot\|_{1}$ for the other cases.

Let $\Gamma$ be a subgroup of a maximal torus $T$ of $G$. Let $\mathcal{L}_{\Gamma}$ be the lattice given by $\Gamma$-invariant weights. The mentioned geometric condition satisfied by the multiplicities of the spherical representations, allows us to give explicit formulas for mult $\lambda_{k}\left(\Delta_{\Gamma}\right)$ in terms of the number of weights in $\mathcal{L}_{\Gamma}$ with a fixed norm (Theorem 3.4). More precisely, in terms of $N_{\mathcal{L}_{\Gamma}}(k):=\#\{\mu \in$ $\left.\mathcal{L}_{\Gamma}:\|\mu\|=k\right\}$. We define in (3.17) the generating theta function associated to the lattice $\mathcal{L}_{\Gamma}$ given by

$$
\vartheta_{\mathcal{L}_{\Gamma}}(z)=\sum_{k \geq 0} N_{\mathcal{L}_{\Gamma}}(k) z^{k}
$$


In Theorem 3.6 we prove

$$
F_{\Delta_{\Gamma}}(z)= \begin{cases}\frac{1}{(1-z)^{n}} \vartheta_{\mathcal{L}_{\Gamma}}(z) & \text { if } M=P^{n}(\mathbb{C}), \\ \frac{1+z}{\left(1-z^{2}\right)^{n}} \vartheta_{\mathcal{L}_{\Gamma}}(z) & \text { if } M=S^{2 n} \\ \frac{1+z}{\left(1-z^{2}\right)^{2}} \vartheta_{D_{2} \cap \mathcal{L}_{\Gamma}}(z) & \text { if } M=P^{1}(\mathbb{H}), \\ \frac{1}{\left(1-z^{2}\right)^{n-1}} \vartheta_{\mathcal{L}_{\Gamma}}(z) & \text { if } M=S^{2 n-1}\end{cases}
$$

Here, $D_{2}=\left\{(a, b) \in \mathbb{Z}^{2}: a+b \equiv 0(\bmod 2)\right\}$. This formula is an alternative and simple way to describe the spectrum of the Laplace-Beltrami operator $\Delta_{\Gamma}$ on $\Gamma \backslash M$, for $M=P^{n}(\mathbb{C}), S^{2 n}$, $P^{1}(\mathbb{H})$ and $S^{2 n-1}$ for $\Gamma$ included in $T$.

Clearly, (1.7) gives an isospectral characterization among locally symmetric spaces of the form $\Gamma \backslash M$ with $M$ as above. Namely, $\Gamma \backslash M$ and $\Gamma^{\prime} \backslash M$ are isospectral if and only if $\vartheta_{\mathcal{L}_{\Gamma}}(z)=\vartheta_{\mathcal{L}_{\Gamma^{\prime}}}(z)$ (Theorem 4.2).

We actually consider in all previous results, Laplace operators twisted by a character of the fundamental group. The spectrum of such operator has a similar description as in the previous case (trivial character). Its associated set $\mathcal{L}_{\Gamma, \chi}$ is in this case a shifted lattice of $\mathcal{L}_{\Gamma}$ and formula (1.7) is still valid.

Furthermore, in the untwisted case (i.e. $\chi$ trivial), we write the generating theta function $\vartheta_{\mathcal{L}_{\Gamma}}(z)$ as a rational function, by using Ehrhart's theory for counting integer points in polytopes (Theorem 3.9). This implies that $F_{\Delta_{\Gamma}}(z)$ is a rational function. We also give in Proposition 3.12 an alternative description of $\vartheta_{\mathcal{L}_{\Gamma}}(z)$ for lens spaces $\Gamma \backslash S^{2 n-1}$, which implies a formula for $F_{\Delta_{\Gamma}}(z)$ already known by Ikeda (see Remark [3.13).

1.3. Examples. In Section 4 we find twisted and untwisted isospectral examples by making use of the isospectral characterization in Theorem 4.2 and the explicit parametrization of cyclic subgroups in $G$ (see 92.3 , \$3.4), where $M=G / K$ is one of the cases considered. By using the computer, we found for small values of $n$ and $q$, every pair of twisted and untwisted isospectral orbifolds covered by $P^{n}(\mathbb{C}), S^{2 n}$ and $S^{2 n-1}$, and also by $P^{1}(\mathbb{H})$ for $n=2$, having cyclic fundamental groups of order $q$.

The most significant examples can be summarized in the next observations:

(1) there exist twisted isospectral lens spaces in dimension 3 (Remark 4.7);

(2) there exist no untwisted isospectral orbifolds covered by $S^{3}$ and $S^{4}$ with cyclic fundamental group of order $q \leq 200$ (Remark 4.7);

(3) there exist untwisted isospectral orbifold lens spaces covered by $S^{d}$ with $5 \leq d \leq 8$, thus such examples exists for any dimension $d \geq 5$ by Sh11] (Remark 4.5);

(4) there exists a 3-dimensional lens space (a manifold) that is twisted isospectral to an orbifold lens space with non-trivial singularities (Remark 4.9);

(5) there are examples of twisted and untwisted isospectrality covered by $P^{n}(\mathbb{C})$ for $n=2,3$, that is, in dimension 4 and 6 ;

(6) there exists a pair of untwisted isospectral orbifolds covered by $P^{1}(\mathbb{H})$ with cyclic fundamental group of order $q=4$, the one with minimum dimension (Example 4.14).

1.4. Previous results. Lens spaces have been a fertile ground for spectral inverse problems. Ikeda and Yamamoto [Y79] YY80] proved that there are no isospectral lens spaces in dimension 3 by giving an explicit formula for the spectral generating function $F_{\Delta_{\Gamma}}(z)$ by using Molien's 
formula. Boldt Bo15] proved that there are no Dirac isospectral 3-dimensional lens spaces with fundamental group of order prime. His proof uses $p$-adic numbers of cyclotomic fields and the explicit formula for the spectral generating functions associated to the positive and negative eigenvalues of the Dirac operator given by Bär Bä96]. Furthermore, Ikeda used the mentioned explicit formula for $F_{\Delta_{\Gamma}}(z)$ to construct several examples (from dimension 5 on) of isospectral lens spaces $([\mathrm{Ik} 80 \mathrm{a}])$. Generalizing such formula for the Hodge-Laplace operators on $p$-forms, he also obtained for each $p_{0}>0$, examples of lens spaces that are $p$-isospectral for every $0 \leq p<p_{0}$ but not $p_{0}$-isospectral ([k88]). Shams Sh11] generalizes Ikeda's method to orbifold lens spaces, obtaining examples from any dimension greater than 8 .

As we mentioned above, in [LMR15], Miatello, Rossetti and the author prove the characterization in Theorem 4.2 for lens spaces. Furthermore, it shows a similar characterization for lens spaces $p$-isospectral for all $p$, which allows them to construct the first pair of Riemannian manifolds $p$-isospectral for all $p$ but not strongly isospectral. DeFord and Doyle [DD14], answering a question in [LMR15], obtain a sufficient condition to construct such examples. In [BL14], Boldt and the author generalize the method in [LMR15] to the Dirac operator on spin lens spaces. The article LMR16 contains a summary of all these results.

Similarly as in Theorem [3.9, Mohades and Honari MH16 have recently used Ehrhart's theory for counting points in rational polytopes to describe the spectrum of a lens space. They also associate to each lens space a toric variety, obtaining geometric consequences for pairs of untwisted isospectral lens spaces.

1.5. Acknowledgement. The author wishes to thank Leandro Cagliero and Jorge Vargas for helpful conversations on representation theory and also Sebastian Boldt and Ramiro Lafuente for helpful comments concerning the algorithms used in the last section. The author also wishes to thank the support of the Oberwolfach Leibniz Fellow programme in May-July 2013 and in August-November 2014, when this project started.

\section{Preliminaries}

This section reviews some of the standard facts on the spectra of twisted Laplace operators on locally symmetric spaces of compact type with cyclic fundamental groups.

2.1. Spectra of twisted Laplace operators. Let $G$ be a Lie group and let $(M, g)$ be a connected compact $G$-homogeneous Riemannian manifold, that is, $G$ acts transitively and almost effectively by isometries on $M$. Let us denote by $K$ the isotropy subgroup of $G$ at some point $p \in M$, and let $\mathfrak{g}$ and $\mathfrak{k}$ be the Lie algebras of $G$ and $K$ respectively. It turns out that $K$ is compact and we have the identifications $M \simeq G / K, g \cdot p \leftrightarrow g K$, and $T_{p} M \simeq T_{e K} G / K \simeq \mathfrak{p}$, where $\mathfrak{p}$ is an $\operatorname{Ad}(K)$-invariant subspace of $\mathfrak{g}$ satisfying $\mathfrak{g}=\mathfrak{k} \oplus \mathfrak{p}$. It is well known that the $G$-invariant Riemannian metrics on $M$ are in correspondence with the $\operatorname{Ad}(K)$-invariant inner products on $\mathfrak{p}$.

We will restrict our attention to the standard metric, that is, the Riemannian metric is given by a negative multiple of the Killing form $B(\cdot, \cdot)$ restricted to $\mathfrak{p} \times \mathfrak{p}$. Note that in this case $G$ must be compact and semisimple. However, we will work in this section under the slightly weaker assumption described in the next paragraph.

We assume that $G$ is a compact Lie group (not necessarily semisimple). Let $\mathfrak{g}$ denote its Lie algebra, thus $\mathfrak{g}=\mathfrak{z} \oplus \mathfrak{g}_{1}$ with $\mathfrak{z}$ the center of $\mathfrak{g}$ and $\mathfrak{g}_{1}=[\mathfrak{g}, \mathfrak{g}]$ the semisimple part. Let $\langle\cdot, \cdot\rangle$ be an inner product on $\mathfrak{g}$ satisfying

- $\left\langle\mathfrak{z}, \mathfrak{g}_{1}\right\rangle=0$;

$\left.\bullet\langle\cdot, \cdot\rangle\right|_{\mathfrak{g}_{1} \times \mathfrak{g}_{1}}=-r B_{\mathfrak{g}_{1}}$ for some $r>0$;

- there is an orthonormal free basis of $\{Z \in \mathfrak{z}: \exp Z=e\}$. 
We assume that the Riemannian metric $g$ on $M$ corresponds to $\left.\langle\cdot, \cdot\rangle\right|_{\mathfrak{p} \times \mathfrak{p} \text {. }}$

Clearly, every discrete subgroup $\Gamma$ in $G$ is finite. The space $\Gamma \backslash M$ inherits a structure of Riemannian (good) orbifold, which is a Riemannian manifold when $\Gamma$ acts freely on $M$ (see for instance Go12]).

Let $\Gamma$ be a finite subgroup of $G$ and let $\chi: \Gamma \rightarrow \mathrm{GL}\left(W_{\chi}\right)$ be a finite dimensional representation of $\Gamma$. We consider the bundle

$$
E_{\Gamma, \chi}:=\Gamma \backslash G \times_{\chi} W_{\chi} \rightarrow \Gamma \backslash G / K
$$

given by the direct product $G \times W_{\chi}$ under the relation $(g, w) \sim(\gamma g k, \chi(\gamma) w)$ for every $k \in K$ and $\gamma \in \Gamma$. We will denote by $[x, w]$ the class of $(x, w) \in G \times W_{\chi}$ in $E_{\Gamma, \chi}$. The space $\Gamma^{\infty}\left(E_{\Gamma, \chi}\right)$ of smooth sections of $E_{\Gamma, \chi}$ is isomorphic to $C^{\infty}(\Gamma \backslash G / K ; \chi):=\left\{f: G \rightarrow W_{\chi}\right.$ smooth : $f(\gamma x k)=$ $\chi(\gamma) f(x) \forall \gamma \in \Gamma, k \in K\}$. Indeed, the function $x K \mapsto[x, f(x)]$ is in $\Gamma^{\infty}\left(E_{\Gamma, \chi}\right)$ if and only if $f \in C^{\infty}(\Gamma \backslash G / K ; \chi)$.

The Lie algebra $\mathfrak{g}$ of $G$ acts on $C^{\infty}(\Gamma \backslash G / K, \chi)$ by

$$
(Y \cdot f)(x)=\left.\frac{d}{d t}\right|_{t=0} f(\exp (t Y) x) .
$$

This action induces a representation of the universal enveloping algebra $U(\mathfrak{g})$ of $\mathfrak{g}$. The Casimir element Cas $\in U(\mathfrak{g})$ is given by Cas $=\sum_{i} X_{i}^{2} \in U(\mathfrak{g})$ where $X_{1}, \ldots, X_{n}$ is any orthonormal basis of $\mathfrak{g}$. The element - Cas induces a self-adjoint elliptic differential operator $\Delta_{M, \Gamma, \chi}$ of second degree on $C^{\infty}(\Gamma \backslash G / K ; \chi)$ and therefore on $\Gamma^{\infty}\left(E_{\Gamma, \chi}\right)$.

Definition 2.1. The operator $\Delta_{M, \Gamma, \chi}$ acting on the smooth section on $E_{\Gamma, \chi}$ is called the $\chi$ twisted Laplace operator. When $\chi=1_{\Gamma}$ (the trivial representation of $\Gamma$ ), $\Delta_{M, \Gamma}:=\Delta_{M, \Gamma, \chi}$ is called the untwisted Laplace operator or just the Laplace operator.

When no confusion can arise on $M$, we just write $\Delta_{\Gamma, \chi}$ and $\Delta_{\Gamma}$ in place of $\Delta_{M, \Gamma, \chi}$ and $\Delta_{M, \Gamma}$ respectively.

Our goal in this subsection is to describe $\operatorname{Spec}(\Gamma \backslash M, \chi)$, the spectrum of $\Delta_{\Gamma, \chi}$. This is given by the multiset of eigenvalues $0<\lambda_{1}<\lambda_{2}<\ldots$ of $\Delta_{\Gamma, \chi}$ with their corresponding multiplicities mult $_{\Delta_{\Gamma, \chi}}\left(\lambda_{k}\right)$ for $k \geq 1$.

Let $\mathfrak{t}_{\mathbb{C}}$ be a Cartan subalgebra of $\mathfrak{g}_{\mathbb{C}}$ and fix a positive system $\Sigma^{+}\left(\mathfrak{g}_{\mathbb{C}}, \mathfrak{t}_{\mathbb{C}}\right)$ in the corresponding root system. Let us denote by $\widehat{G}$ the unitary dual of $G$, that is, the class of (finite dimensional) unitary irreducible representations of $G$. It is well known that the Casimir element Cas acts by a scalar on each $\pi \in \widehat{G}$ (see for instance [Wa73, Lemma 5.6.4]). We denote by $\lambda(C a s, \pi)$ the opposite of this scalar, so

$$
\lambda(\text { Cas, } \pi):=\left\langle\Lambda_{\pi}+\rho, \Lambda_{\pi}+\rho\right\rangle-\langle\rho, \rho\rangle,
$$

where $\Lambda_{\pi}$ is the highest weight of $\pi$ and $\rho$ denotes half the sum of the positive roots relative to $\Sigma^{+}\left(\mathfrak{g}_{\mathbb{C}}, \mathfrak{t}_{\mathbb{C}}\right)$.

Notation 2.2. For an arbitrary closed subgroup group $H$ of $G$ and $\tau_{1}, \tau_{2}$ representations of $H$, set $\left[\tau_{1}: \tau_{2}\right]=\operatorname{dim} \operatorname{Hom}_{H}\left(\tau_{1}, \tau_{2}\right)$. For example: if $\tau_{1}$ is irreducible, then $\left[\tau_{1}: \tau_{2}\right]$ is the number of times that $\tau_{1}$ appears in the decomposition into irreducibles of $\tau_{2}$; if $1_{H}$ denotes the trivial representation of $H$ and $\pi$ is a representation of $G$, then $\left[1_{H}:\left.\pi\right|_{H}\right]=\operatorname{dim} V_{\pi}^{H}$, the dimension of the subspace of $H$-invariants in $V_{\pi}$. For $\lambda \in \mathbb{R}$, we set

$$
\begin{aligned}
\widehat{G}_{H} & =\left\{\pi \in \widehat{G}:\left[1_{H}:\left.\pi\right|_{H}\right]>0\right\}, \\
\widehat{G}(\lambda) & =\{\pi \in \widehat{G}: \lambda(\text { Cas }, \pi)=\lambda\}, \\
\widehat{G}_{H}(\lambda) & =\widehat{G}_{H} \cap \widehat{G}(\lambda) .
\end{aligned}
$$


The elements in $\widehat{G}_{H}$ are usually called the $H$-spherical representations of $G$.

The next result describes the spectrum of $\Delta_{\Gamma, \chi}$ in terms of $\chi$-invariants of $K$-spherical representations of $G$ restricted to $\Gamma$.

Theorem 2.3. Let $M=G / K$ be a compact homogeneous manifold with a Riemannian metric as above, let $\Gamma$ be a finite subgroup of $G$ and let $\chi$ be a finite dimensional representation of $\Gamma$. The set of eigenvalues in $\operatorname{Spec}(\Gamma \backslash M, \chi)$ is contained in

$$
\mathcal{E}(M):=\left\{\lambda(\text { Cas, } \pi): \pi \in \widehat{G}_{K}\right\} .
$$

Moreover, the multiplicity of $\lambda \in \mathcal{E}(M)$ in $\operatorname{Spec}(\Gamma \backslash M, \chi)$ is given by

$$
\operatorname{mult}_{\Delta_{\Gamma, \chi}}(\lambda):=\sum_{\pi \in \widehat{G}_{K}(\lambda)}\left[\chi:\left.\pi\right|_{\Gamma}\right]\left[1_{K}:\left.\pi\right|_{K}\right] .
$$

In other words, the spectral zeta function of $\Delta_{\Gamma, \chi}$ is given by

$$
\zeta_{M, \Gamma, \chi}(s)=\sum_{\pi \in \widehat{G}_{K}}\left[\chi:\left.\pi\right|_{\Gamma}\right]\left[1_{K}:\left.\pi\right|_{K}\right] \lambda(C a s, \pi)^{-s} .
$$

Proof. This result is well known, we sketch the proof for completeness. We consider, on the Hilbert space $L^{2}(G)$, the representation of $G \times G$ by $L_{g_{1}} \times R_{g_{2}}$ where $\left(L_{g} \cdot f\right)(x)=f\left(g^{-1} x\right)$ and $\left(R_{g} \cdot f\right)(x)=f(x g)$ for $f \in L^{2}(G)$. The Peter-Weyl Theorem implies the decomposition

$$
L^{2}(G) \simeq \bigoplus_{\pi \in \widehat{G}} V_{\pi} \otimes V_{\pi}^{*}
$$

as $G \times G$-modules. Here, $v \otimes \varphi \in V_{\pi} \otimes V_{\pi}^{*}$ induces the function $f_{v \otimes \varphi}(x):=\varphi\left(\pi(x)^{-1} \cdot v\right)$, therefore the action of $G \times G$ on each component $V_{\pi} \otimes V_{\pi}^{*}$ is given by $\left(g_{1}, g_{2}\right) \cdot(v \otimes \varphi)=$ $\left(\pi\left(g_{1}\right) \cdot v\right) \otimes\left(\pi^{*}\left(g_{2}\right) \cdot \varphi\right)$.

The subspace of $L^{2}$-functions on $G$ invariant by $K$ on the right is

$$
L^{2}(M)=L^{2}(G / K) \simeq \bigoplus_{\pi \in \widehat{G}} V_{\pi} \otimes\left(V_{\pi}^{*}\right)^{K} .
$$

On the other hand, we have the map $\Psi: C^{\infty}(G / K) \otimes W_{\chi} \rightarrow C^{\infty}\left(G / K, W_{\chi}\right):=\{\psi: G \rightarrow$ $W_{\chi}$ smooth : $\left.\psi(g k)=\psi(g) \forall g \in G, k \in K\right\}$, given by $\Psi(f, w)(x)=f(x) w$. The group $\Gamma$ acts on $C^{\infty}(G / K)$ by the restriction of the action $L_{g}$ of $G$. One can check that the map $\Psi$ sends the subspace of $\Gamma$-invariants elements $\left(C^{\infty}(G / K) \otimes W_{\chi}\right)^{\Gamma}$ onto $C^{\infty}(\Gamma \backslash G / K ; \chi)$. Hence, $\Gamma^{\infty}\left(E_{\Gamma, \chi}\right) \simeq\left(C^{\infty}(G / K) \otimes W_{\chi}\right)^{\Gamma}$. By taking the closure in the corresponding spaces, we obtain that

$$
\begin{aligned}
L^{2}\left(E_{\Gamma, \chi}\right) & \simeq\left(L^{2}(G / K) \otimes W_{\chi}\right)^{\Gamma} \simeq \bigoplus_{\pi \in \widehat{G}}\left(V_{\pi} \otimes W_{\chi}\right)^{\Gamma} \otimes\left(V_{\pi}^{*}\right)^{K} \\
& \simeq \bigoplus_{\pi \in \widehat{G}} \operatorname{Hom}_{\Gamma}\left(W_{\chi}, V_{\pi}^{*}\right) \otimes\left(V_{\pi}^{*}\right)^{K}=\bigoplus_{\pi \in \widehat{G}_{K}} \operatorname{Hom}_{\Gamma}\left(W_{\chi}, V_{\pi}\right) \otimes V_{\pi}^{K} .
\end{aligned}
$$

The Casimir element Cas also acts on $L^{2}(G / K)$ leaving invariant each term in (2.6). Moreover, this action commutes with the left action of $G$ on $C^{\infty}(G / K)$, in particular with elements in $\Gamma$, thus $\Delta_{\Gamma, \chi}$ acts on each term $\operatorname{Hom}_{\Gamma}\left(W_{\chi}, V_{\pi}\right) \otimes V_{\pi}^{K}$ by the scalar $\lambda($ Cas, $\pi)$. This proves that any eigenvalue of $\Delta_{\Gamma, \chi}$ is $\lambda($ Cas,$\pi)$ for some $\pi \in \widehat{G}_{K}$. Moreover, since $\operatorname{dim} \operatorname{Hom}_{\Gamma}\left(W_{\chi}, V_{\pi}\right)=\left[\chi:\left.\pi\right|_{\Gamma}\right]$ and $\operatorname{dim} V_{\pi}^{K}=\left[1_{K}:\left.\pi\right|_{K}\right]$, (2.7) implies the formula for mult $\Delta_{\Gamma, \chi}(\lambda)$.

The formula for the spectral zeta function follows immediately from the previous description. This concludes the proof. 
The previous theorem says that in order to describe $\operatorname{Spec}(\Gamma \backslash M, \chi)$ one has to calculate three ingredients:

- $\widehat{G}_{K}$ (with the corresponding numbers $\left[1_{K}:\left.\pi\right|_{K}\right]$ for $\pi \in \widehat{G}_{K}$ );

- the numbers $\left[\chi:\left.\pi\right|_{\Gamma}\right]$ for $\pi \in \widehat{G}_{K}$;

- the numbers $\lambda($ Cas, $\pi)$ for $\pi \in \widehat{G}_{K}$.

The last one can be easily done by (2.1). The first one is the most complicated for arbitrary $(G, K)$, though it is well known in our particular cases of interest, namely, compact symmetric spaces of real rank one. We next deal with the second one, under the assumption of $\Gamma$ is included in a maximal torus $T$ of $G$, taking in mind the situation of $\Gamma$ cyclic.

2.2. Abelian fundamental group. We associate a maximal torus of $G$ to the Cartan subalgebra $\mathfrak{t}_{\mathbb{C}}$ of $\mathfrak{g}_{\mathbb{C}}$ as usual. Let $\mathfrak{t}_{\mathbb{R}}=\left\{X \in \mathfrak{t}_{\mathbb{C}}: \alpha(X) \in \mathbb{R} \forall \alpha \in \Sigma\left(\mathfrak{g}_{\mathbb{C}}, \mathfrak{t}_{\mathbb{C}}\right)\right\}$, and let $T$ be the connected subgroup of $G$ with Lie algebra $\mathfrak{t}:=i \mathfrak{t}_{\mathbb{R}}$, thus $T$ is a maximal torus in $G$. We denote by $P\left(\mathfrak{g}_{\mathbb{C}}\right)$ the set of weights of $\mathfrak{g}_{\mathbb{C}}\left(\right.$ i.e. $\mu \in \mathfrak{t}_{\mathbb{C}}^{*}$ such that $2\langle\mu, \alpha\rangle /\langle\alpha, \alpha\rangle \in \mathbb{Z}$ for all $\alpha \in \Sigma\left(\mathfrak{g}_{\mathbb{C}}, \mathfrak{t}_{\mathbb{C}}\right)$ ); by $P(G)$ the subset of $\mu \in P\left(\mathfrak{g}_{\mathbb{C}}\right)$ such that there exists $\xi: T \rightarrow \mathbb{C}^{\times}$with $\xi(\exp (X))=e^{\mu(X)}$ for all $X \in \mathfrak{t}$; by $P^{++}(\mathfrak{g})$ the dominant weights in $P(\mathfrak{g})$ and $P^{++}(G)=P(G) \cap P^{++}(\mathfrak{g})$. For $g \in T$ and $\mu \in P(G)$ let $g^{\mu}=e^{\mu(X)}$ for any $X \in \mathfrak{t}$ satisfying $\exp X=g$.

We assume that $\Gamma$ is contained in $T$, thus $\Gamma$ is abelian, so its irreducible representations are one-dimensional (i.e. characters).

Lemma 2.4. Let $\Gamma$ be a finite subgroup of $T$. If $\chi: \Gamma \rightarrow \mathbb{C}^{\times}$is a morphism and $\pi$ is a finite dimensional representation of $G$, then $\left[\chi:\left.\pi\right|_{\Gamma}\right]=\operatorname{dim} V_{\pi}^{\chi(\Gamma)}$, where

$$
V_{\pi}^{\chi(\Gamma)}=\left\{v \in V_{\pi}: \pi(\gamma) \cdot v=\chi(\gamma) v \forall \gamma \in \Gamma\right\} .
$$

Proof. Since $\Gamma \subset T$, the weight space $V_{\pi}(\mu)=\left\{v \in V_{\pi}: \pi(g) \cdot v=g^{\mu} v \quad \forall g \in T\right\}$ is invariant by $\Gamma$ for any $\mu \in P(G)$. Moreover, as a representation of $\Gamma, V_{\pi}(\mu)$ is equivalent to $m_{\pi}(\mu)$ copies of the character $\gamma \mapsto \gamma^{\mu}$, where $m_{\pi}(\mu):=\operatorname{dim} V_{\pi}(\mu)$ is the multiplicity of $\mu$ in $\pi$. Hence, the subrepresentation $V_{\pi}(\mu)$ of $\Gamma$ contains $\chi$ if and only if $\chi(\gamma)=\gamma^{\mu}$ for every $\gamma \in \Gamma$, and in this case $\left[\chi:\left.V_{\pi}(\mu)\right|_{\Gamma}\right]=m_{\pi}(\mu)$. The proof follows since $V_{\pi}$ is a direct sum of its weight spaces.

Definition 2.5. Let $\Gamma$ be a finite subgroup of $T$ and let $\chi: \Gamma \rightarrow \mathbb{C}^{\times}$be a character. We associate to $\Gamma$ and $\chi$ the affine congruence lattice

$$
\mathcal{L}_{\Gamma, \chi}:=\left\{\mu \in P(G): \gamma^{\mu}=\chi(\gamma) \quad \forall \gamma \in \Gamma\right\} .
$$

If $\chi=1_{\Gamma}$, we call it just the congruence lattice associated to $\Gamma$ and denote it by $\mathcal{L}_{\Gamma}$.

One can easily see that $\mathcal{L}_{\Gamma}$ is a sublattice of $P(G)$ and $\mathcal{L}_{\Gamma, \chi}$ is a shifted lattice of $\mathcal{L}_{\Gamma}$, that is, $\mathcal{L}_{\Gamma, \chi}=\mu+\mathcal{L}_{\Gamma}$ for any $\mu \in \mathcal{L}_{\Gamma, \chi}$.

Proposition 2.6. Let $\Gamma$ be a finite subgroup of $T$ and let $\chi: \Gamma \rightarrow \mathbb{C}^{\times}$be a character. For each representation $\pi$ of $G$ we have that

$$
\left[\chi:\left.\pi\right|_{\Gamma}\right]=\operatorname{dim} V_{\pi}^{\chi(\Gamma)}=\sum_{\mu \in \mathcal{L}_{\Gamma, \chi}} m_{\pi}(\mu)
$$

Proof. We decompose $V_{\pi}=\oplus_{\mu \in P(G)} V_{\pi}(\mu)$ into weight spaces. Let $v=\sum_{\mu \in P(G)} v_{\mu} \in V_{\pi}$ with $v_{\mu} \in V_{\pi}(\mu)$ for every $\mu \in P(G)$. Clearly, $v_{\mu} \neq 0$ for only finitely many $\mu$. We claim that $v \in V_{\pi}^{\chi(\Gamma)}$ if and only if $v_{\mu} \in V_{\pi}^{\chi(\Gamma)}$ for every $\mu \in P(G)$. Indeed, if $v \in V_{\pi}^{\chi(\Gamma)}$ then

$$
\sum_{\mu \in P(G)} \chi(\gamma) v_{\mu}=\chi(\gamma) v=\pi(\gamma) \cdot v=\sum_{\mu \in P(G)} \pi(\gamma) \cdot v_{\mu}=\sum_{\mu \in P(G)} \gamma^{\mu} v_{\mu}
$$


for every $\gamma \in \Gamma$, thus $\chi(\gamma)=\gamma^{\mu}$ for every $\mu \in P(G)$ such that $v_{\mu} \neq 0$, hence $v_{\mu} \in V_{\pi}(\mu)^{\chi(\Gamma)}$ for every $\mu \in P(G)$. The converse follows immediately. We obtain that

$$
V_{\pi}^{\chi(\Gamma)}=\bigoplus_{\mu \in P(G)} V_{\pi}(\mu)^{\chi(\Gamma)}
$$

By (2.9),

$$
V_{\pi}(\mu)^{\chi(\Gamma)}= \begin{cases}V_{\pi}(\mu) & \text { if } \mu \in \mathcal{L}_{\Gamma, \chi} \\ 0 & \text { if } \mu \notin \mathcal{L}_{\Gamma, \chi}\end{cases}
$$

Hence

$$
\operatorname{dim} V_{\pi}^{\chi(\Gamma)}=\sum_{\mu \in P(G)} \operatorname{dim} V_{\pi}(\mu)^{\chi(\Gamma)}=\sum_{\mu \in \mathcal{L}_{\Gamma, \chi}} \operatorname{dim} V_{\pi}(\mu)=\sum_{\mu \in \mathcal{L}_{\Gamma, \chi}} m_{\pi}(\mu)
$$

as asserted.

2.3. Cyclic subgroups of classical groups. In this subsection we first fix the root system associated to each classical compact group. The conventions used are the standard ones, so the reader can skip this part coming back to it for notation when necessary. We also classify their cyclic subgroups up to conjugation.

- Tyре $A_{n}$. Here $G=\mathrm{SU}(n+1), \mathfrak{g}=\mathfrak{s u}(n+1), \mathfrak{g}_{\mathbb{C}}=\mathfrak{s l}(n+1, \mathbb{C})$,

$$
\begin{aligned}
T & =\left\{\operatorname{diag}\left(e^{\mathrm{i} \theta_{1}}, \ldots, e^{\mathrm{i} \theta_{n+1}}\right): \theta_{i} \in \mathbb{R} \forall i, \sum_{i} \theta_{i}=0\right\}, \\
\mathfrak{t}_{\mathbb{C}} & =\left\{\operatorname{diag}\left(\theta_{1}, \ldots, \theta_{n+1}\right): \theta_{i} \in \mathbb{C} \forall i, \quad \sum_{i} \theta_{i}=0\right\},
\end{aligned}
$$

$\varepsilon_{i}\left(\operatorname{diag}\left(\theta_{1}, \ldots, \theta_{n+1}\right)\right)=\theta_{i}$ for each $1 \leq i \leq n+1, \Sigma^{+}\left(\mathfrak{g}_{\mathbb{C}}, \mathfrak{t}_{\mathbb{C}}\right)=\left\{\varepsilon_{i}-\varepsilon_{j}: i<j\right\}, \Pi\left(\mathfrak{g}_{\mathbb{C}}, \mathfrak{t}_{\mathbb{C}}\right)=$ $\left\{\varepsilon_{i}-\varepsilon_{i+1}: 1 \leq i \leq n\right\}, \rho=\sum_{i=1}^{n+1} \frac{n-2 i+2}{2} \varepsilon_{i}$, and

$$
\begin{aligned}
P(G) & =\left\{\sum_{i} a_{i} \varepsilon_{i} \in \mathbb{Z} \varepsilon_{1} \oplus \cdots \oplus \mathbb{Z} \varepsilon_{n+1}: \sum_{i} a_{i}=0\right\}, \\
P^{++}(G) & =\left\{\sum_{i} a_{i} \varepsilon_{i} \in P(G): a_{1} \geq a_{2} \geq \cdots \geq a_{n+1}\right\} .
\end{aligned}
$$

- Type $B_{n}$. Here $G=\mathrm{SO}(2 n+1), \mathfrak{g}=\mathfrak{s o}(2 n+1), \mathfrak{g}_{\mathbb{C}}=\mathfrak{s o}(2 n+1, \mathbb{C})$,

$$
\begin{aligned}
T & =\left\{\operatorname{diag}\left(\left[\begin{array}{cc}
\cos \left(\theta_{1}\right) & \sin \left(\theta_{1}\right) \\
-\sin \left(\theta_{1}\right) & \cos \left(\theta_{1}\right)
\end{array}\right], \ldots,\left[\begin{array}{cc}
\cos \left(\theta_{n}\right) & \sin \left(\theta_{n}\right) \\
-\sin \left(\theta_{n}\right) & \cos \left(\theta_{n}\right)
\end{array}\right], 1\right): \theta_{i} \in \mathbb{R} \forall i\right\}, \\
\mathfrak{t}_{\mathbb{C}} & =\left\{\operatorname{diag}\left(\left[\begin{array}{cc}
0 & \theta_{1} \\
-\theta_{1} & 0
\end{array}\right], \ldots,\left[\begin{array}{cc}
0 & \theta_{n} \\
-\theta_{n} & 0
\end{array}\right], 0\right): \theta_{i} \in \mathbb{C} \forall i\right\},
\end{aligned}
$$

$\varepsilon_{i}\left(\operatorname{diag}\left(\left[\begin{array}{cc}0 & \theta_{1} \\ -\theta_{1} & 0\end{array}\right], \ldots,\left[\begin{array}{cc}0 & \theta_{n} \\ -\theta_{n} & 0\end{array}\right], 0\right)\right)=\theta_{i}$ for each $1 \leq i \leq n, \Sigma^{+}\left(\mathfrak{g}_{\mathbb{C}}, \mathfrak{t}_{\mathbb{C}}\right)=\left\{\varepsilon_{i} \pm \varepsilon_{j}: i<j\right\} \cup\left\{\varepsilon_{i}\right\}$, $\Pi\left(\mathfrak{g}_{\mathbb{C}}, \mathfrak{t}_{\mathbb{C}}\right)=\left\{\varepsilon_{i}-\varepsilon_{i+1}: 1 \leq i \leq n-1\right\} \cup\left\{\varepsilon_{n}\right\}, \rho=\sum_{i=1}^{n}\left(n-\frac{2 i-1}{2}\right) \varepsilon_{i}, P(G)=\mathbb{Z} \varepsilon_{1} \oplus \cdots \oplus \mathbb{Z} \varepsilon_{n}$, and

$$
P^{++}(G)=\left\{\sum_{i} a_{i} \varepsilon_{i} \in P(G): a_{1} \geq a_{2} \geq \cdots \geq a_{n} \geq 0\right\} .
$$

- Type $C_{n}$. Here $G=\operatorname{Sp}(n, \mathbb{C}) \cap \mathrm{U}(2 n)$ where $\operatorname{Sp}(n, \mathbb{C})=\left\{g \in \operatorname{SL}(2 n, \mathbb{C}): g^{t} J_{n} g=J_{n}:=\right.$ $\left.\left(\begin{array}{cc}0 & \operatorname{Id}_{n} \\ -\operatorname{Id}_{n} & 0\end{array}\right)\right\}, \mathfrak{g}=\mathfrak{s p}(n, \mathbb{C}) \cap \mathfrak{u}(2 n), \mathfrak{g}_{\mathbb{C}}=\mathfrak{s p}(2 n, \mathbb{C})$,

$$
\begin{aligned}
T & =\left\{\operatorname{diag}\left(e^{\mathrm{i} \theta_{1}}, \ldots, e^{\mathrm{i} \theta_{n}}, e^{-\mathrm{i} \theta_{1}}, \ldots, e^{-\mathrm{i} \theta_{n}}\right): \theta_{i} \in \mathbb{R} \forall i\right\}, \\
\mathfrak{t}_{\mathbb{C}} & =\left\{\operatorname{diag}\left(\theta_{1}, \ldots, \theta_{n},-\theta_{1}, \ldots,-\theta_{n}\right): \theta_{i} \in \mathbb{C} \forall i\right\},
\end{aligned}
$$

$\varepsilon_{i}\left(\operatorname{diag}\left(\theta_{1}, \ldots, \theta_{n},-\theta_{1}, \ldots,-\theta_{n}\right)\right)=\theta_{i}$ for each $1 \leq i \leq n, \Sigma^{+}\left(\mathfrak{g}_{\mathbb{C}}, \mathfrak{t}_{\mathbb{C}}\right)=\left\{\varepsilon_{i} \pm \varepsilon_{j}: 1 \leq i<j \leq\right.$ $n\} \cup\left\{2 \varepsilon_{i}: 1 \leq i \leq n\right\}, \Pi\left(\mathfrak{g}_{\mathbb{C}}, \mathfrak{t}_{\mathbb{C}}\right)=\left\{\varepsilon_{i}-\varepsilon_{i+1}: 1 \leq i \leq n-1\right\} \cup\left\{2 \varepsilon_{n}\right\}, \rho=\sum_{i=1}^{n}(n-i+1) \varepsilon_{i}$, $P(G)=\mathbb{Z} \varepsilon_{1} \oplus \cdots \oplus \mathbb{Z} \varepsilon_{n}$

$$
P^{++}(G)=\left\{\sum_{i} a_{i} \varepsilon_{i} \in P(G): a_{1} \geq \cdots \geq a_{n-1} \geq a_{n} \geq 0\right\} .
$$


- Type $D_{n}$. Here $G=\mathrm{SO}(2 n), \mathfrak{g}=\mathfrak{s o}(2 n), \mathfrak{g}_{\mathbb{C}}=\mathfrak{s o}(2 n, \mathbb{C})$,

$$
\begin{aligned}
T & =\left\{\operatorname{diag}\left(\left[\begin{array}{cc}
\cos \left(\theta_{1}\right) & \sin \left(\theta_{1}\right) \\
-\sin \left(\theta_{1}\right) & \cos \left(\theta_{1}\right)
\end{array}\right], \ldots,\left[\begin{array}{cc}
\cos \left(\theta_{n}\right) & \sin \left(\theta_{n}\right) \\
-\sin \left(\theta_{n}\right) & \cos \left(\theta_{n}\right)
\end{array}\right]\right): \theta_{i} \in \mathbb{R} \forall i\right\}, \\
\mathfrak{t}_{\mathbb{C}} & =\left\{\operatorname{diag}\left(\left[\begin{array}{cc}
0 & \theta_{1} \\
-\theta_{1} & 0
\end{array}\right], \ldots,\left[\begin{array}{cc}
0 & \theta_{n} \\
-\theta_{n} & 0
\end{array}\right]\right): \theta_{i} \in \mathbb{C} \forall i\right\},
\end{aligned}
$$

$\varepsilon_{i}\left(\operatorname{diag}\left(\left[\begin{array}{cc}0 & \theta_{1} \\ -\theta_{1} & 0\end{array}\right], \ldots,\left[\begin{array}{cc}0 & \theta_{n} \\ -\theta_{n} & 0\end{array}\right]\right)\right)=\theta_{i}$ for each $1 \leq i \leq n, \Sigma^{+}\left(\mathfrak{g}_{\mathbb{C}}, \mathfrak{t}_{\mathbb{C}}\right)=\left\{\varepsilon_{i} \pm \varepsilon_{j}: i<j\right\}$, $\Pi\left(\mathfrak{g}_{\mathbb{C}}, \mathfrak{t}_{\mathbb{C}}\right)=\left\{\varepsilon_{i}-\varepsilon_{i+1}: 1 \leq i \leq n-1\right\} \cup\left\{\varepsilon_{n-1}+\varepsilon_{n}\right\}, \rho=\sum_{i=1}^{n}(n-i) \varepsilon_{i}, P(G)=\mathbb{Z} \varepsilon_{1} \oplus \cdots \oplus \mathbb{Z} \varepsilon_{n}$,

$$
P^{++}(G)=\left\{\sum_{i} a_{i} \varepsilon_{i} \in P(G): a_{1} \geq \cdots \geq a_{n-1} \geq\left|a_{n}\right|\right\} \text {. }
$$

We now classify the cyclic subgroups in the classical compact groups. Let $G$ be either $\mathrm{SU}(n+1), \mathrm{SO}(2 n+1), \mathrm{Sp}(n)$ or $\mathrm{SO}(2 n)$, thus the maximal torus $T$ of $G$ fixed above has dimension $n$. We consider a cyclic subgroup $\Gamma$ of $G$. Since any element in $G$ is conjugate to an element in $T$, we can assume that $\Gamma$ is included in the maximal torus. Note that for any closed subgroup $K$ of $G$, the locally homogeneous spaces $\Gamma \backslash G / K$ and $\left(g^{-1} \Gamma g\right) \backslash G / K$ are isometric for any $g \in G$.

For $q \in \mathbb{N}$ and $s=\left(s_{1}, \ldots, s_{n}\right) \in \mathbb{Z}^{n}$, we set

$$
\gamma_{q, s}= \begin{cases}\operatorname{diag}\left(\xi_{q}^{s_{1}}, \ldots, \xi_{q}^{s_{n}}, \xi_{q}^{-\left(s_{1}+\cdots+s_{n}\right)}\right) & \text { if } G=\operatorname{SU}(n+1), \\ \operatorname{diag}\left(R\left(\frac{2 \pi s_{1}}{q}\right), \ldots, R\left(\frac{2 \pi s_{n}}{q}\right), 1\right) & \text { if } G=\operatorname{SO}(2 n+1), \\ \operatorname{diag}\left(\xi_{q}^{s_{1}}, \ldots, \xi_{q}^{s_{n}}, \xi_{q}^{-s_{1}}, \ldots, \xi_{q}^{-s_{n}}\right) & \text { if } G=\operatorname{Sp}(n), \\ \operatorname{diag}\left(R\left(\frac{2 \pi s_{1}}{q}\right), \ldots, R\left(\frac{2 \pi s_{n}}{q}\right)\right) & \text { if } G=\operatorname{SO}(2 n),\end{cases}
$$

where $\xi_{q}=e^{2 \pi i / q}$ and $R(\theta)=\left(\begin{array}{cc}\cos \theta & \sin \theta \\ -\sin \theta & \cos \theta\end{array}\right)$.

Notation 2.7. For $s \in \mathbb{Z}^{n}$, write $s_{n+1}=-\left(s_{1}+\cdots+s_{n}\right)$. This notation simplifies the case $G=\mathrm{SU}(n+1)$. The notation $\operatorname{gcd}(q, s)$ means $\operatorname{gcd}\left(q, s_{1}, \ldots, s_{n+1}\right)$ for $G=\mathrm{SU}(n+1)$ or $\operatorname{gcd}\left(q, s_{1}, \ldots, s_{n}\right)$ in the rest of the cases.

Let $q \in \mathbb{N}$ and $s \in \mathbb{Z}^{n}$ satisfying $\operatorname{gcd}(q, s)=1$. Clearly, $\gamma_{q, s}$ is in $T$ and has order $q$. Moreover, any finite subgroup of $T$ of order $q$ is equal to $\Gamma_{q, s}:=\left\langle\gamma_{q, s}\right\rangle$ for some $s \in \mathbb{Z}^{n}$. The matrix $\gamma_{q, s}$ has eigenvalues $\left\{\xi^{s_{1}}, \ldots, \xi^{s_{n+1}}\right\}$ for $G=\mathrm{SU}(n+1),\left\{\xi_{q}^{ \pm s_{1}}, \ldots, \xi_{q}^{ \pm s_{n}}, 1\right\}$ for $G=\mathrm{SO}(2 n+1)$, and $\left\{\xi_{q}^{ \pm s_{1}}, \ldots, \xi_{q}^{ \pm s_{n}}\right\}$ for $G=\operatorname{Sp}(n), \operatorname{SO}(2 n)$.

When $G=\operatorname{SO}(2 n)$ and $\operatorname{gcd}\left(s_{j}, q\right)=1$ for all $j$, the space $\Gamma_{q, s} \backslash S^{2 n-1} \simeq \Gamma_{q, s} \backslash \operatorname{SO}(2 n) / \operatorname{SO}(2 n-$ $1)$ is a lens space. It is denoted by $L\left(q ; s_{1}, \ldots, s_{n}\right)$ in Ikeda's papers and in [LMR15]. Lens spaces are the only manifolds of constant sectional curvature with cyclic fundamental group. If the condition $\operatorname{gcd}\left(s_{j}, q\right)=1$ does not hold for some $j$, then $\gamma_{q, s}^{q / \operatorname{gcd}\left(s_{j}, q\right)}$ is a non-trivial element in $\Gamma_{q, s}$ having the eigenvalue 1 , thus $\Gamma_{q, s}$ does not act freely on $S^{2 n-1}$. Hence, $\Gamma_{q, s} \backslash S^{2 n-1}$ is an orbifold with (non-trivial) singularities, so it is not a manifold. Such orbifolds were called orbifold lens spaces in [Sh11].

Proposition 2.8. Let $G=\mathrm{SU}(n+1), q \in \mathbb{N}$ and $s, s^{\prime} \in \mathbb{Z}^{n}$ such that $\operatorname{gcd}(q, s)=\operatorname{gcd}\left(q, s^{\prime}\right)=1$. The groups $\Gamma_{q, s}$ and $\Gamma_{q, s^{\prime}}$ are conjugate in $G$ if and only if there exist $\sigma$ a permutation of $\{1, \ldots, n+1\}$ and $\ell \in \mathbb{Z}$ coprime to $q$ satisfying

$$
s_{\sigma(j)} \equiv \ell s_{j}^{\prime} \quad(\bmod q) \quad \text { for all } 1 \leq j \leq n+1 .
$$

Proof. The cyclic subgroups $\Gamma_{q, s}$ and $\Gamma_{q, s^{\prime}}$ are conjugate in $G$ if and only if there exists an integer $\ell$ coprime to $q$ such that $\gamma_{q, s}$ and $\gamma_{q, s^{\prime}}^{\ell}$ are conjugate in $G$. This condition holds if and only if $\gamma_{q, s}$ and $\gamma_{q, s^{\prime}}^{\ell}$ have the same multiset of eigenvalues, which is equivalent to $s_{\sigma(j)} \equiv \ell s_{j}^{\prime}$ $(\bmod q)$ for all $j$, for some permutation $\sigma$. 
Proposition 2.9. Let $G$ be either $\mathrm{SO}(2 n+1), \operatorname{Sp}(n)$ or $\mathrm{SO}(2 n)$. Let $q \in \mathbb{N}$ and $s, s^{\prime} \in \mathbb{Z}^{n}$ such that $\operatorname{gcd}(q, s)=\operatorname{gcd}\left(q, s^{\prime}\right)=1$. The groups $\Gamma_{q, s}$ and $\Gamma_{q, s^{\prime}}$ are conjugate in $G$ if and only if there are $\sigma$ a permutation of $\{1, \ldots, n\}, \epsilon_{1}, \ldots, \epsilon_{n} \in\{ \pm 1\}$, and $\ell \in \mathbb{Z}$ coprime to $q$ satisfying

$$
s_{\sigma(j)} \equiv \epsilon_{j} \ell s_{j}^{\prime} \quad(\bmod q) \quad \text { for all } 1 \leq j \leq n .
$$

Proof. Similarly as above, $\Gamma_{q, s}$ and $\Gamma_{q, s^{\prime}}$ are conjugate in $G$ if and only if $\gamma_{q, s}$ and $\gamma_{q, s^{\prime}}^{\ell}$ have the same eigenvalues for some integer $\ell$ coprime to $q$, which is equivalent to

$$
\left\{\xi_{q}^{ \pm s_{1}}, \ldots, \xi_{q}^{ \pm s_{n}}\right\}=\left\{\xi_{q}^{ \pm \ell s_{1}^{\prime}}, \ldots, \xi_{q}^{ \pm \ell s_{n}^{\prime}}\right\}
$$

The proof thus follows.

\section{Description OF SPECTRA}

We mentioned at the end of $\$ 2.1$ that the most difficult ingredient to compute $\operatorname{Spec}(\Gamma \backslash G / K, \chi)$ is the determination of the $K$-spherical representations $\widehat{G}_{K}$. In $\$ 3.1$ we consider compact symmetric spaces of real rank one because $\widehat{G}_{K}$ is very simple in these case. We next describe the spectrum of their corresponding orbifolds in two ways. First, we give a formula for the multiplicity of the $k$-th eigenvalue in $\$ 3.2$, and secondly, we give in $\$ 3.3$ a formula for their corresponding generating function. We conclude the section by computing the generating functions for cyclic subgroups.

3.1. Symmetric spaces of real rank one. For a fuller treatment on symmetric spaces, including their classification, we refer the reader to [He2]. See also [He1, Ch. V] for a description of the spectra of compact symmetric spaces.

Let $M$ be a compact symmetric space of real rank one. Since we are interested in their quotients, we assume that $M$ is simply connected, so real projective spaces are excluded. In what follows, we fix the realization of $M=G / K$ as:

\begin{tabular}{cccc}
$G$ & $K$ & $M$ & $\operatorname{dim} M$ \\
\hline $\mathrm{SU}(n+1)$ & $\mathrm{S}(\mathrm{U}(n) \times \mathrm{U}(1))$ & $P^{n}(\mathbb{C})$ & $2 n$ \\
$\mathrm{SO}(2 n+1)$ & $\mathrm{SO}(2 n)$ & $S^{2 n}$ & $2 n$ \\
$\mathrm{Sp}(n)$ & $\mathrm{Sp}(n-1) \times \mathrm{Sp}(1)$ & $P^{n-1}(\mathbb{H})$ & $4 n-4$ \\
$\mathrm{SO}(2 n)$ & $\mathrm{SO}(2 n-1)$ & $S^{2 n-1}$ & $2 n-1$ \\
$\mathrm{~F}_{4}$ & $\mathrm{Spin}(9)$ & $P^{2}(\mathbb{O})$ & 16
\end{tabular}.

In all classical cases, we consider the Riemannian metric $g_{\text {can }}$ on $M$ induced by

$$
\langle X, Y\rangle=\frac{1}{2} \operatorname{Tr}\left(X^{*} Y\right) .
$$

It is well known that $\left(S^{n}, g_{\text {can }}\right)$ has constant sectional curvature one.

Following the notation introduced in $\oint 2.3$, let $\Lambda_{0} \in P(G)$ given by

$$
\Lambda_{0}= \begin{cases}\varepsilon_{1}-\varepsilon_{n+1} & \text { for } G=\operatorname{SU}(n+1), \\ \varepsilon_{1} & \text { for } G=\operatorname{SO}(2 n+1), \\ \varepsilon_{1}+\varepsilon_{2} & \text { for } G=\operatorname{Sp}(n), \\ \varepsilon_{1} & \text { for } G=\operatorname{SO}(2 n), \\ \varepsilon_{1} & \text { for } G=\mathrm{F}_{4} .\end{cases}
$$

It is well known that

$$
\widehat{G}_{K}=\left\{\pi_{k \Lambda_{0}}: k \in \mathbb{Z}_{\geq 0}\right\}
$$


Moreover, $\left[1_{K}:\left.\pi\right|_{K}\right]=1$ for every $\pi \in \widehat{G}_{K}$. In order to write Theorem 2.3 in this particular case, let $\pi_{k}=\pi_{k \Lambda_{0}}$ and $\lambda_{k}=\lambda\left(\right.$ Cas, $\left.\pi_{k}\right)$, thus $\mathcal{E}(M)=\left\{\lambda_{k}: k \geq 0\right\}$ where

$$
\lambda_{k}= \begin{cases}k(k+n) & \text { if } M=P^{n}(\mathbb{C}), \\ k(k+2 n-1) & \text { if } M=S^{2 n}, \\ k(k+2 n-1) & \text { if } M=P^{n-1}(\mathbb{H}), \\ k(k+2 n-2) & \text { if } M=S^{2 n-1}, \\ k(k+11) & \text { if } M=P^{2}(\mathbb{O}) .\end{cases}
$$

Theorem 3.1. Let $M=G / K$ be a simply connected compact Riemannian symmetric space of real rank one, let $\Gamma$ be a finite subgroup of $G$ and let $\chi$ be a finite dimensional representation of $\Gamma$. Then, every eigenvalue in $\operatorname{Spec}(\Gamma \backslash M, \chi)$ is in $\left\{\lambda_{k}: k \geq 0\right\}$ and $\operatorname{mult}_{\Delta_{M, \Gamma, \chi}}\left(\lambda_{k}\right):=\left[\chi:\left.\pi_{k}\right|_{\Gamma}\right]$ for all $k \geq 0$. Equivalently,

$$
\zeta_{M, \Gamma, \chi}(s)=\sum_{k \geq 0}\left[\chi:\left.\pi_{k}\right|_{\Gamma}\right] \lambda_{k}^{-s}
$$

3.2. Multiplicity formula. By Theorem [3.1, the multiplicity mult $_{\Delta_{M, \Gamma, \chi}}\left(\lambda_{k}\right)=\left[\chi:\left.\pi_{k}\right|_{\Gamma}\right]$ is the only remaining ingredient for a given $(\Gamma, \chi)$ to describe $\operatorname{Spec}(\Gamma \backslash M, \chi)$. We will determine it under the assumption that the discrete subgroup $\Gamma$ is contained in the maximal torus of $G$, situation already considered in \$2.2.

Let us denote by $T$ the maximal torus of $G$ fixed in 92.3 . Assume that $\Gamma$ is a finite subgroup of $T$, thus mult ${ }_{\Delta_{M, \Gamma, \chi}}\left(\lambda_{k}\right)=\left[\chi,\left.\pi_{k}\right|_{\Gamma}\right]$ by Theorem 3.1. Proposition 2.6 now yields

$$
\operatorname{mult}_{\Delta_{M, \Gamma, \chi}}\left(\lambda_{k}\right)=\sum_{\mu \in \mathcal{L}_{\Gamma, \chi}} m_{\pi_{k}}(\mu) .
$$

The next goal is to calculate the multiplicities $m_{\pi_{k}}(\mu)$. There exist several formulas for them, though there do not exist many 'closed' formulas as we require.

As it is usual, for $\mu=\sum_{i} a_{i} \varepsilon_{i} \in P(G)$ we denote

$$
\|\mu\|_{1}=\sum_{i}\left|a_{i}\right| \quad \text { and } \quad\|\mu\|_{\infty}=\max _{i}\left(\left|a_{i}\right|\right)
$$

the one-norm and the maximum norm of $\mu$ respectively. In the literature, $\|\cdot\|_{1}$ is also known as $l^{1}$-norm or taxicab norm, and $\|\cdot\|_{\infty}$ as $l^{\infty}$-norm or uniform norm. We will usually work with $\|\cdot\|_{\infty}$ only when $G=\operatorname{Sp}(2)$ and with $\|\cdot\|_{1}$ when $G$ is either $\operatorname{SU}(n+1), \operatorname{SO}(2 n+1)$ or $\operatorname{SO}(2 n)$ for $n \geq 2$. We will sometimes write $\|\cdot\|$ for $\|\cdot\|_{1}$ or $\|\cdot\|_{\infty}$ depending on $G$ as above. We will use the notation $\lfloor x\rfloor$ for the largest integer smaller than or equal to $x$.

Proposition 3.2. Let $k$ be a non-negative integer. If $G=\mathrm{SU}(n+1)$ and $\mu=\sum_{i=1}^{n+1} a_{i} \varepsilon_{i} \in P(G)$, then

$$
m_{\pi_{k}}(\mu)= \begin{cases}\left(\begin{array}{c}
k-\frac{1}{2}\|\mu\|_{1}+n-1 \\
n-1
\end{array}\right) & \text { if } k-\frac{1}{2}\|\mu\|_{1} \in \mathbb{Z}_{\geq 0}, \\
0 & \text { otherwise. }\end{cases}
$$

If $G=\mathrm{SO}(2 n+1)$ and $\mu=\sum_{i=1}^{n} a_{i} \varepsilon_{i} \in P(G)$, then

$$
m_{\pi_{k}}(\mu)= \begin{cases}\left(\begin{array}{c}
\left\lfloor\frac{1}{2}\left(k-\|\mu\|_{1}\right)\right\rfloor+n-1 \\
n-1
\end{array}\right) & \text { if } k-\|\mu\|_{1} \in \mathbb{Z}_{\geq 0}, \\
0 & \text { otherwise. }\end{cases}
$$


If $G=\operatorname{Sp}(2)$ and $\mu=\sum_{i=1}^{2} a_{i} \varepsilon_{i} \in P(G)$, then

$$
m_{\pi_{k}}(\mu)= \begin{cases}\left\lfloor\frac{1}{2}\left(k-\|\mu\|_{\infty}\right)\right\rfloor+1 & \text { if } k-\|\mu\|_{\infty} \in \mathbb{Z}_{\geq 0}, \text { and } a_{1}+a_{2} \in 2 \mathbb{Z}, \\ 0 & \text { otherwise. }\end{cases}
$$

If $G=\mathrm{SO}(2 n)$ and $\mu=\sum_{i=1}^{n} a_{i} \varepsilon_{i} \in P(G)$, then

$$
m_{\pi_{k}}(\mu)= \begin{cases}\left(\begin{array}{c}
\frac{1}{2}\left(k-\|\mu\|_{1}\right)+n-2 \\
n-2
\end{array}\right) & \text { if } k-\|\mu\|_{1} \in 2 \mathbb{Z}_{\geq 0}, \\
0 & \text { otherwise. }\end{cases}
$$

Proof. Formula (3.12) has been already proved in [LMR15, Lem. 3.6], and (3.10) follows in a very similar way. Formula (3.11) is a particular case of the general formula in [CT04, Thm. 4.1].

We now suppose that $G=\mathrm{SU}(n+1)$. Probably this case is already proved in the literature, but we could not find it stated in the form above. We can assume that $\mu=\sum_{i=1}^{n+1} a_{i} \varepsilon_{i}$ is dominant, thus $a_{1} \geq a_{2} \geq \cdots \geq a_{n} \geq a_{n+1}$ since the Weyl group in this case is the permutation group in $n+1$ letters, which preserves $\|\cdot\|_{1}$. Suppose that $r:=k-\frac{1}{2}\|\mu\|_{1} \geq 0$, thus $\|\mu\|_{1} \leq\|\Lambda\|_{1}$. The highest weight $\Lambda_{k}=k \varepsilon_{1}-k \varepsilon_{n+1}$ and the dominant weight $\mu$ have associated partitions

$$
\begin{aligned}
\Lambda_{k} & \rightsquigarrow k(n+1)=2 k+\underbrace{k+\cdots+k}_{n-1}+0, \\
\mu & \rightsquigarrow k(n+1)=\left(a_{1}+k\right)+\cdots+\left(a_{n+1}+k\right) .
\end{aligned}
$$

Hence, $m_{\pi_{k}}(\mu)$ is equal to the number of ways one can fill the Young diagram associated to $\Lambda_{k}$ with $a_{1}+k 1$ 's, $a_{2}+k 2$ 's, $\ldots, a_{n+1}+k(n+1)$ 's, in such a way that the entries in each row are non-decreasing and those in each column are strictly increasing (see for instance [FH, page 224]). This number is known as the Kostka number of $\mu$ in $\Lambda_{k}$, that is, the number of semistandard tableaux on $\Lambda_{k}$ of type $\mu$. The corresponding Young diagram to $\Lambda_{k}$ has $2 k$ boxes in the first row and $k$ boxes in the next $n-1$ rows. The remaining combinatorial computation is left to the reader.

Remark 3.3. The previous lemma tell us that $m_{\pi_{k}}(\mu)$ depends only on $\|\mu\|_{1}$ when $G$ is either $\mathrm{SU}(n+1), \mathrm{SO}(2 n+1)$ or $\mathrm{SO}(2 n)$. If $G=\mathrm{Sp}(2)$, by restricting $\mu$ to the lattice

$$
D_{2}:=\left\{\left(a_{1}, a_{2}\right) \in \mathbb{Z}^{2}: a_{1}+a_{2} \in 2 \mathbb{Z}\right\}
$$

we obtain that $\operatorname{mult}_{\pi_{k}}(\mu)$ depends only on $\|\mu\|_{\infty}$.

One can check that for $G=\operatorname{Sp}(n)$ with $n \geq 3$ and $G=\mathrm{F}_{4}$ the multiplicity formula mult $\pi_{k}(\mu)$ cannot be written as above. An even more complicated situation appears when one considers a compact symmetric space of real rank greater than one since the set of spherical representations cannot be written as in (3.4). This reason forces us to restrict our attention to orbifolds covered by spheres, complex projective spaces and the quaternion projective line.

For any $k \geq 0$ and for any subset $\mathcal{L}$ in $P(G)$, let

$$
N_{\mathcal{L}}(k)=\#\{\mu \in \mathcal{L}:\|\mu\|=k\},
$$

where $\|\cdot\|$ stands for $\|\cdot\|_{1}$ in all cases except for $G=\operatorname{Sp}(2)$ where $\|\cdot\|$ denotes $\|\cdot\|_{\infty}$.

Theorem 3.4. Let $M=G / K$ be either $P^{n}(\mathbb{C}), S^{2 n}, P^{1}(\mathbb{H})$ or $S^{2 n-1}$ with $n \geq 2$, realized as in (3.1). Let $T$ be the maximal torus in $G$ fixed in $\$ 2.3$. Let $\Gamma$ be a (finite) subgroup of $T$, let $\chi: \Gamma \rightarrow \mathbb{C}^{\times}$be a character, and let $\mathcal{L}_{\Gamma, \chi}$ be the associated affine congruence lattice defined in 
Definition 2.5. Then mult ${ }_{\Delta_{M, \Gamma, \chi}}\left(\lambda_{k}\right)$ is given by

$$
\begin{cases}\sum_{r=0}^{k}\left(\begin{array}{c}
r+n-1 \\
n-1
\end{array}\right) N_{\mathcal{L}_{\Gamma, \chi}}(2 k-2 r) & \text { if } M=P^{n}(\mathbb{C}), \\
\sum_{r=0}^{\lfloor k / 2\rfloor}\left(\begin{array}{c}
r+n-1 \\
n-1
\end{array}\right)\left(N_{\mathcal{L}_{\Gamma, \chi}}(k-2 r)+N_{\mathcal{L}}(k-1-2 r)\right) & \text { if } M=S^{2 n}, \\
\sum_{r=0}^{\lfloor k / 2\rfloor}(r+1)\left(N_{\mathcal{L}_{\Gamma, \chi}}(k-2 r)+N_{\mathcal{L}}(k-1-2 r)\right) & \text { if } M=P^{1}(\mathbb{H}), \\
\sum_{r=0}^{\lfloor k / 2\rfloor}\left(\begin{array}{c}
r+n-2 \\
n-2
\end{array}\right) N_{\mathcal{L}_{\Gamma, \chi}}(k-2 r) & \text { if } M=S^{2 n-1} .\end{cases}
$$

Proof. We first assume $M=P^{n}(\mathbb{C})$. From (3.9) we have that $m_{\pi_{k}}(\mu)=0$ if $\|\mu\|_{1}>2 k$, thus

$$
\operatorname{mult}_{\Delta_{\Gamma, \chi}}\left(\lambda_{k}\right)=\sum_{r=0}^{\lfloor k / 2\rfloor} \sum_{\substack{\mu \in \mathcal{L}_{\Gamma, \chi},\|\mu\|_{1}=2 k-2 r}} m_{\pi_{k}}(\mu)
$$

by (3.7) and the fact that $\|\mu\|_{1}$ is always even. Note that $m_{\pi_{k}}(\mu)=\left(\begin{array}{c}r+n-1 \\ n-1\end{array}\right)$ by (3.9) for any $\mu$ satisfying $\|\mu\|_{1}=2 k-2 r$. Since $\left(\begin{array}{c}r+n-1 \\ n-1\end{array}\right)$ does not depend on $\mu$, the inner sum is equal to $\left(\begin{array}{c}r+n-1 \\ n-1\end{array}\right) N_{\mathcal{L}_{\Gamma, \chi}}(2 k-2 r)$, which proves the formula in this case.

We next assume $M=S^{2 n}$. Again, combining (3.7) and (3.10) we obtain that

$$
\text { mult }_{\Delta_{\Gamma, \chi}}\left(\lambda_{k}\right)=\sum_{r=0}^{\lfloor k / 2\rfloor} \sum_{\substack{\mu \in \mathcal{L}_{\Gamma, \chi} \\
\|\mu\|=k-2 r}}\left(\begin{array}{c}
r+n-1 \\
n-1
\end{array}\right)+\sum_{r=0}^{\lfloor(k-1) / 2\rfloor} \sum_{\substack{\mu \in \mathcal{L}_{\Gamma, \mathcal{X}} \\
\|\mu\|=k-1-2 r}}\left(\begin{array}{c}
r+n-1 \\
n-1
\end{array}\right),
$$

which establishes the formula. The other cases follow in a very similar way.

Remark 3.5. The formula for $M=S^{2 n-1}$ and $\chi$ trivial was already obtained in LMR15, Thm. 3.5].

3.3. Generating functions. The spectral zeta function is not very appropriate for explicit computations in our particular spaces. See for instance [CB94a, [CB94b], Sp03, Ha11, [Teo14], BF08, where many technical computations are made to determine its poles, residues and the determinant. We introduce here a generating function encoding the spectrum of an orbifold covered by a complex projective space, a sphere or the quaternion projective line. Generating functions were already used by Ikeda in several papers (IK80b], [Ik80c, [Ik80a], [Ik83], [Ik88], [Ik97], IY79]), obtaining many examples of isospectral spherical space forms and also spectral rigidity results.

We define the spectral generating function associated to $\Delta_{M, \Gamma, \chi}$ or to $\operatorname{Spec}(\Gamma \backslash M, \chi)$, by

$$
F_{\Delta_{M, \Gamma, \chi}}(z):=\sum_{k \geq 0} \operatorname{mult}_{\Delta_{M, \Gamma, \chi}}\left(\lambda_{k}\right) z^{k} .
$$

We shall abbreviate $F_{\Gamma, \chi}(z)$ in place of $F_{\Delta_{M, \Gamma, \chi}}(z)$ when no confusion can arise. We also write $F_{\Gamma}(z)$ when $\chi=1_{\Gamma}$. 
For any subset $\mathcal{L}$ in $P(G)$, we define the generating theta function associated to $\mathcal{L}$ by

$$
\vartheta_{\mathcal{L}}(z)= \begin{cases}\sum_{k \geq 0} N_{\mathcal{L}}(2 k) z^{k} & \text { if } G=\mathrm{SU}(n+1), \\ \sum_{k \geq 0} N_{\mathcal{L}}(k) z^{k} & \text { if } G=\mathrm{SO}(2 n+1), \operatorname{Sp}(2), \mathrm{SO}(2 n) .\end{cases}
$$

The number $N_{\mathcal{L}}(k)$ was defined in (3.14) and depends on the norm $\|\cdot\|$ on $P(G)$. Note that $\|\mu\|_{1}$ is even for any $\mu \in P(G)$ when $G=\mathrm{SU}(n+1)$.

Theorem 3.6. Under the same hypotheses of Theorem 3.4, we have that

$$
F_{\Gamma, \chi}(z)= \begin{cases}\frac{1}{(1-z)^{n}} \vartheta_{\mathcal{L}_{\Gamma, \chi}}(z) & \text { if } M=P^{n}(\mathbb{C}), \\ \frac{1+z}{\left(1-z^{2}\right)^{n}} \vartheta_{\mathcal{L}_{\Gamma, \chi}}(z) & \text { if } M=S^{2 n}, \\ \frac{1+z}{\left(1-z^{2}\right)^{2}} \vartheta_{D_{2} \cap \mathcal{L}_{\Gamma, \chi}}(z) & \text { if } M=P^{1}(\mathbb{H}), \\ \frac{1}{\left(1-z^{2}\right)^{n-1}} \vartheta_{\mathcal{L}_{\Gamma, \chi}}(z) & \text { if } M=S^{2 n-1} .\end{cases}
$$

Proof. Assume $M=P^{n}(\mathbb{C})$, thus $G=\mathrm{SU}(n+1)$. Theorem 3.4 implies that

$$
\begin{aligned}
F_{\Gamma, \chi}(z) & =\sum_{k \geq 0}\left(\sum_{r=0}^{k}\left(\begin{array}{c}
r+n-1 \\
n-1
\end{array}\right) N_{\mathcal{L}_{\Gamma, \chi}}(2 k-2 r)\right) z^{k} \\
& =\left(\sum_{k \geq 0}\left(\begin{array}{c}
k+n-1 \\
n-1
\end{array}\right) z^{k}\right)\left(\sum_{k \geq 0} N_{\mathcal{L}_{\Gamma, \chi}}(2 k) z^{k}\right) \\
& =\frac{1}{(1-z)^{n}} \vartheta_{\mathcal{L}_{\Gamma, \chi}}(z) .
\end{aligned}
$$

The rest of the cases are also a direct consequence of Theorem 3.4 and follow in a very similar way.

3.4. Cyclic fundamental group case. We next describe the associated affine congruence lattice $\mathcal{L}_{\Gamma, \chi}$ for $\Gamma=\Gamma_{q, s}$ as in $\oint 2.3$, for $M=P^{n}(\mathbb{C}), S^{2 n}, P^{1}(\mathbb{H}), S^{2 n-1}$. We also write the corresponding generating theta function as a rational function in the untwisted case (i.e. $\chi$ trivial).

Let $q \in \mathbb{N}$ and $s \in \mathbb{Z}^{n}$ such that $\operatorname{gcd}(q, s)=1$ (recall the convention in Notation 2.7). Since $\Gamma_{q, s}$ is cyclic and generated by $\gamma_{q, s}$ as in (2.23), any character $\chi: \Gamma_{q, s} \rightarrow \mathbb{C}^{\times}$is determined by the value of $\chi\left(\gamma_{q, s}\right)$, which is a $q$-root of unity. When $q$ and $s$ are fixed, for $u \in \mathbb{Z}$, we denote by $\chi_{u}$ the character of $\Gamma_{q, s}$ such that $\chi_{u}\left(\gamma_{q, s}\right)=\xi_{q}^{u}$. Clearly, $\left\{\chi_{u}: 0 \leq u<q\right\}$ is a representative set of $\widehat{\Gamma}_{q, s}$.

Proposition 3.7. Let $q \in \mathbb{N}, s \in \mathbb{Z}^{n}$ and $u \in \mathbb{Z}$ such that $\operatorname{gcd}(q, s)=1$. Then

$$
\mathcal{L}_{\Gamma_{q, s}, \chi_{u}}=\left\{\sum_{i} a_{i} \varepsilon_{i} \in P(G): \sum_{i} a_{i} s_{i} \equiv u \quad(\bmod q)\right\} .
$$

Here, the summation runs over $1 \leq i \leq n+1$ for $G=\mathrm{SU}(n+1)$ and over $1 \leq i \leq n$ for $G=\mathrm{SO}(2 n+1), \operatorname{Sp}(n), \mathrm{SO}(2 n)$. 
Proof. By definition, $\mathcal{L}_{\Gamma_{q, s}, \chi_{u}}=\left\{\mu \in P(G): \gamma_{q, s}^{\mu}=\xi_{q}^{u}\right\}$ since $\Gamma_{q, s}$ is generated by $\gamma_{q, s}$. Let

$$
H_{q, s}= \begin{cases}\operatorname{diag}\left(2 \pi i s_{1} / q, \ldots, 2 \pi i s_{n+1} / q\right) & \text { if } G=\operatorname{SU}(n+1), \\
\operatorname{diag}\left(\left(\begin{array}{cc}
0 & 2 \pi s_{1} / q \\
-2 \pi s_{1} / q & 0
\end{array}\right), \ldots,\left(\begin{array}{cc}
0 & 2 \pi s_{n} / q \\
-2 \pi s_{n} / q & 0
\end{array}\right), 0\right) & \text { if } G=\operatorname{SO}(2 n+1), \\
\operatorname{diag}\left(2 \pi i s_{1} / q, \ldots, 2 \pi i s_{n} / q,-2 \pi i s_{1} / q, \ldots,-2 \pi i s_{n} / q\right) & \text { if } G=\operatorname{Sp}(n), \\
\operatorname{diag}\left(\left(\begin{array}{cc}
0 & 2 \pi s_{1} / q \\
-2 \pi s_{1} / q & 0
\end{array}\right), \ldots,\left(\begin{array}{cc}
0 & 2 \pi s_{n} / q \\
-2 \pi s_{n} / q & 0
\end{array}\right)\right) & \text { if } G=\operatorname{SO}(2 n),\end{cases}
$$

thus $\exp \left(H_{q, s}\right)=\gamma_{q, s}$ in all cases. Hence, $\gamma_{q, s}^{\mu}=e^{\mu\left(H_{q, s}\right)}$ and, since

$$
\mu\left(H_{q, s}\right)=\sum_{i} a_{i} \varepsilon_{i}\left(H_{q, s}\right)=\sum_{i} a_{i} \frac{2 \pi i s_{i}}{q}=\frac{2 \pi \mathrm{i}}{q} \sum_{i} a_{i} s_{i}
$$

for $\mu=\sum_{i} a_{i} \varepsilon_{i} \in P(G)$, we obtain that $\gamma_{q, s}^{\mu}=\xi_{q}^{u}$ if and only if $\sum_{i} a_{i} s_{i} \equiv u(\bmod q)$. This completes the proof.

Notation 3.8. Let $A_{n}=\left\{\left(a_{1}, \ldots, a_{n+1}\right) \in \mathbb{Z}^{n+1}: a_{1}+\cdots+a_{n+1}=0\right\}$. We will make use of the obvious correspondences $P(\mathrm{SU}(n+1)) \simeq A_{n}$ and $P(G) \simeq \mathbb{Z}^{n}$ for $G=\mathrm{SO}(2 n), \operatorname{Sp}(n), \operatorname{SO}(2 n+1)$ given by $\sum_{i} a_{i} \varepsilon_{i} \leftrightarrow\left(a_{1}, a_{2}, \ldots\right)$. For $q \in \mathbb{N}, s \in \mathbb{Z}^{n}$ and $u \in \mathbb{Z}$ as above, let

$$
\begin{aligned}
& \mathcal{L}_{q, s, u}^{*}=\left\{\left(a_{1}, \ldots, a_{n+1}\right) \in A_{n}: \sum_{i=1}^{n+1} a_{i} s_{i} \equiv u \quad(\bmod q)\right\}, \\
& \mathcal{L}_{q, s, u}=\left\{\left(a_{1}, \ldots, a_{n}\right) \in \mathbb{Z}^{n}: \sum_{i=1}^{n} a_{i} s_{i} \equiv u \quad(\bmod q)\right\} .
\end{aligned}
$$

Thus, $\mathcal{L}_{\Gamma_{q, s}, \chi_{u}}$ corresponds to $\mathcal{L}_{q, s, u}^{*}$ or to $\mathcal{L}_{q, s, u}$ depending on $G=\mathrm{SU}(n+1)$ or $G=\operatorname{SO}(2 n+$ $1), \operatorname{Sp}(n), \mathrm{SO}(2 n)$ respectively. We write $\mathcal{L}_{q, s}^{*}=\mathcal{L}_{q, s, 0}^{*}$ and $\mathcal{L}_{q, s}=\mathcal{L}_{q, s, 0}$.

One must be careful with the notation $\mathcal{L}_{q, s, u}$ since it works for $G=\mathrm{SO}(2 n+1), \mathrm{Sp}(2), \mathrm{SO}(2 n)$, but $\|\cdot\|$ on $P(G)$ coincides with $\|\cdot\|_{1}$ when $G=\operatorname{SO}(2 n+1), \operatorname{SO}(2 n)$ and with $\|\cdot\|_{\infty}$ when $G=\operatorname{Sp}(2)$. However, by Theorem 3.6, one can distinguish their corresponding generating theta function as $\vartheta_{\mathcal{L}_{q, s, u}}(z)$ for $G=\mathrm{SO}(2 n+1), \mathrm{SO}(2 n)$ and $\vartheta_{D_{2} \cap \mathcal{L}_{q, s, u}}(z)$ for $G=\operatorname{Sp}(2)$.

Our next goal is, fixing $u=0$ (i.e. $\chi_{u}$ is the trivial character), to give formulas for the generating theta function associated to $\mathcal{L}_{q, s}^{*}, \mathcal{L}_{q, s}$ and $D_{2} \cap \mathcal{L}_{q, s}$. Consequently we will obtain that the spectral generating function of an orbifold having cyclic fundamental group covered by complex projective spaces, spheres and the quaternion projective line is a rational function.

We start by writing the theta function as a rational function by using Ehrhart's theory for counting points in rational polytopes. For more details on Ehrhart's theory see the nice book [BR].

Theorem 3.9. Let $q \in \mathbb{N}$ and $s \in \mathbb{Z}^{n}$ such that $\operatorname{gcd}(q, s)=1$. Let $\mathcal{L}$ be either $\mathcal{L}_{q, s}^{*}, \mathcal{L}_{q, s}$ for any $n \geq 2$ or $D_{2} \cap \mathcal{L}_{q, s}$ for $n=2$. Then, there exist a polynomial $p(z)$ of degree less than $(n+1) q$ such that

$$
\vartheta_{\mathcal{L}}(z)=\frac{(1-z) p(z)}{\left(1-z^{q}\right)^{n+1}}
$$

Proof. Let

$$
\mathcal{B}= \begin{cases}\left\{x \in \mathbb{R}^{n+1}:\|x\|_{1} \leq 2, \sum_{i=1}^{n+1} x_{i}=0\right\} & \text { if } \mathcal{L}=\mathcal{L}_{q, s}^{*}, \\ \left\{x \in \mathbb{R}^{n}:\|x\|_{1} \leq 1\right\} & \text { if } \mathcal{L}=\mathcal{L}_{q, s}, \\ \left\{x \in \mathbb{R}^{2}:\|x\|_{\infty} \leq 1\right\} & \text { if } \mathcal{L}=D_{2} \cap \mathcal{L}_{q, s} .\end{cases}
$$


Note that $\mathcal{B}$ is a cross-polytope in $\mathbb{R}^{n}$ in the second case and a cube in $\mathbb{R}^{2}$ in the third case, both centered at the origin. Clearly, $N_{\mathcal{L}}(k)$ is the number of points in $\mathcal{L}$ at the $k^{\text {th }}$ dilate of the boundary of $\mathcal{B}$, that is, $N_{\mathcal{L}}(k)=\#(\mathcal{L} \cap k \partial \mathcal{B})$. Hence

$$
\vartheta_{\mathcal{L}}(z)=\sum_{k \geq 0} \#(\mathcal{L} \cap k \partial \mathcal{B}) z^{k}=(1-z) \sum_{k \geq 0} \#(\mathcal{L} \cap k \mathcal{B}) z^{k} .
$$

Let $\Psi$ be a linear transformation sending the canonical basis in $\mathbb{R}^{n}$ to a basis of $\mathcal{L}$. Let $\mathcal{P}=\Psi(\mathcal{B})$, hence

$$
\vartheta_{\mathcal{L}}(z)=(1-z) \sum_{k \geq 0} \#\left(\mathbb{Z}^{n} \cap k \mathcal{P}\right) z^{k} .
$$

The function $\operatorname{Ehr}_{\mathcal{P}}(z):=\sum_{k \geq 0} \#\left(\mathbb{Z}^{n} \cap k \mathcal{P}\right) z^{k}$ is called the Ehrhart series of $\mathcal{P}$. By the Ehrhart Theorem for rational polytopes (see [BR, §3.7]), there exists a polynomial $p(z)$ of degree less than $q(n+1)$ such that $\operatorname{Ehr}_{\mathcal{P}}(z)=p(z) /\left(1-z^{q}\right)^{n+1}$, which establishes the formula.

The above theorem implies that the map $\Phi(k):=\sum_{m=0}^{k} N_{\mathcal{L}}(m)$, which sends $k$ to the $k^{\text {th }}$ coefficient of the series $\vartheta_{\mathcal{L}}(z) /(1-z)$, is a quasipolynomial on $k$ of degree $n$ and its period divides $q$ (see [BR, Thm. 3.23]). The next remark gives the coefficients of $p(z)$ in terms of the coefficients of $\vartheta_{\mathcal{L}}(z)$, which is useful for explicit computations.

Remark 3.10. Write $p(z)=\sum_{j=0}^{q(n+1)-1} h_{j} z^{j}$ the polynomial in the proof of Theorem 3.9. By expanding the expression $p(z)=(1-z)^{-1}\left(1-z^{q}\right)^{n+1} \vartheta_{\mathcal{L}}(z)$ we obtain, for $0 \leq k_{0}<q$ and $0 \leq l_{0} \leq n$, that

$$
h_{k_{0}+l_{0} q}=\sum_{j=0}^{l_{0}}(-1)^{j}\left(\begin{array}{c}
n+1 \\
j
\end{array}\right) \sum_{m=0}^{k_{0}+q\left(l_{0}-j\right)} N_{\mathcal{L}}(m) .
$$

Note that $\sum_{m=0}^{k} N_{\mathcal{L}}(m)=\#\left(\mathbb{Z}^{n} \cap k \mathcal{P}\right)$, the $k$-th coefficient of the Ehrhart series $\operatorname{Ehr}_{\mathcal{P}}(z)$ in the proof of Theorem 3.9.

Remark 3.11. Theorem 3.9 shows that the order of the fundamental group $q$ is a spectral invariant. That is, if two orbifolds with cyclic fundamental group, both covered by $P^{n}(\mathbb{C}), S^{2 n}$, $P^{1}(\mathbb{H})$ or $S^{2 n-1}$, are untwisted isospectral, then their fundamental groups have the same order.

We next show an alternative way to compute $\vartheta_{\mathcal{L}_{q, s}}(z)$.

Proposition 3.12. Let $q \in \mathbb{N}$ and $s \in \mathbb{Z}^{n}$ such that $\operatorname{gcd}(q, s)=1$. Then

$$
\vartheta_{\mathcal{L}_{q, s}}(z)=\frac{\left(1-z^{2}\right)^{n}}{q} \sum_{l=0}^{q-1} \prod_{j=1}^{n} \frac{1}{\left(z-\xi_{q}^{l s_{j}}\right)\left(z-\xi_{q}^{-l_{s_{j}}}\right)} .
$$

Proof. We have

$$
\vartheta_{\mathcal{L}_{q, s}}(z)=\sum_{\mu \in \mathcal{L}_{q, s}} z^{\|\mu\|_{1}}=\sum_{\left(a_{1}, \ldots, a_{n}\right) \in \mathbb{Z}^{n}}\left(\frac{1}{q} \sum_{l=0}^{q-1} \xi_{q}^{l \sum_{i} a_{i} s_{i}}\right) z^{\sum_{i}\left|a_{i}\right|} .
$$

By switching the summations, we obtain that

$$
\vartheta_{\mathcal{L}_{q, s}}(z)=\frac{1}{q} \sum_{l=0}^{q-1} \prod_{j=1}^{n}\left(1+\sum_{m \geq 1} \xi_{q}^{m l s_{j}} z^{m l}+\sum_{m \geq 1} \xi_{q}^{-m l s_{j}} z^{m l}\right) .
$$

Since the quantity inside the parenthesis is equal to $\left(1-z \xi_{q}^{l s_{j}}\right)^{-1}+\left(1-z \xi_{q}^{-l s_{j}}\right)^{-1}-1=(1-$ $\left.z^{2}\right)\left(1-z \xi_{q}^{l s_{j}}\right)^{-1}\left(1-z \xi_{q}^{-l s_{j}}\right)^{-1}$, the assertion follows. 
The previous proof was communicated by Prof. Don Zagier to the author at Oberwolfach in July 2013.

Remark 3.13. This proposition and Theorem 3.6 imply that the generating function associated to the lens space $\Gamma_{q, s} \backslash S^{2 n-1}$ is

$$
F_{\Gamma_{q, s}}(z)=\frac{1-z^{2}}{q} \sum_{l=0}^{q-1} \prod_{j=1}^{n} \frac{1}{\left(z-\xi_{q}^{l s_{j}}\right)\left(z-\xi_{q}^{-l s_{j}}\right)} .
$$

This formula has been already proved in [IY79, Thm. 3.2], and was the main tool for Ikeda's construction of isospectral lens spaces $([\mathrm{Ik} 80 \mathrm{a}])$.

\section{ISOSPECTRALITY}

This section studies the case when two Laplace operators are isospectral, that is, their spectra coincide. Of course, the notion of isospectrality among orbifolds coincides with the equality of their corresponding spectral generating function. Now, Theorem 3.6 in our cases of interest, implies that this notion is equivalent to the coincidence of the corresponding generating theta functions. This characterization is very useful to find examples of twisted and untwisted isospectral orbifolds.

Usually, one says that two orbifolds are isospectral if their corresponding untwisted Laplace operator acting on functions are isospectral. However, in this paper we will always add the word untwisted to avoid confusion. Similarly, we will say that two orbifolds are twisted isospectral if there exist nontrivial twists on each of them so that their corresponding twisted Laplace operators are isospectral.

4.1. Isospectral characterization. Let $G$ be either $\mathrm{SU}(n+1), \mathrm{SO}(2 n+1), \mathrm{Sp}(2)$ or $\mathrm{SO}(2 n)$. Recall that $P(G)$ is identified with $A_{n}, \mathbb{Z}^{n}, \mathbb{Z}^{2}$ and $\mathbb{Z}^{n}$ respectively (see Notation 3.8), and the norm $\|\cdot\|$ on $P(G)$ is $\|\cdot\|_{1}$ excepting for $G=\operatorname{Sp}(2)$ where it is $\|\cdot\|_{\infty}$.

Definition 4.1. Let $\mathcal{L}$ and $\mathcal{L}^{\prime}$ be two subsets of $P(G)$. We say that they are $\|\cdot\|$-isospectral if $\vartheta_{\mathcal{L}}(z)=\vartheta_{\mathcal{L}^{\prime}}(z)$, or equivalently, $N_{\mathcal{L}}(k)=N_{\mathcal{L}^{\prime}}(k)$ for all $k \geq 0$. More precisely,

$$
\#\{\mu \in \mathcal{L}:\|\mu\|=k\}=\#\left\{\mu \in \mathcal{L}^{\prime}:\|\mu\|=k\right\} \quad \forall k \geq 0 .
$$

The next isospectral characterization is a direct consequence of Theorem 3.6.

Theorem 4.2. Let $M=G / K$ be either $P^{n}(\mathbb{C}), S^{2 n}, P^{1}(\mathbb{H})$ or $S^{2 n-1}$ for $n \geq 2$. Let $\Gamma$ and $\Gamma^{\prime}$ be finite subgroups of the maximal torus $T$ of $G$ and let $\chi$ and $\chi^{\prime}$ be characters of $\Gamma$ and $\Gamma^{\prime}$ respectively. If $M \neq P^{1}(\mathbb{H}), \Delta_{\Gamma, \chi}$ and $\Delta_{\Gamma^{\prime}, \chi^{\prime}}$ are isospectral if and only if $\mathcal{L}_{\Gamma, \chi}$ and $\mathcal{L}_{\Gamma^{\prime}, \chi^{\prime}}$ are $\|\cdot\|_{1}$-isospectral. If $M=P^{1}(\mathbb{H}), \Delta_{\Gamma, \chi}$ and $\Delta_{\Gamma^{\prime}, \chi^{\prime}}$ are isospectral if and only if $D_{2} \cap \mathcal{L}_{\Gamma, \chi}$ and $D_{2} \cap \mathcal{L}_{\Gamma^{\prime}, \chi^{\prime}}$ are $\|\cdot\|_{\infty}$-isospectral.

Proof. We have that $\Delta_{\Gamma, \chi}$ and $\Delta_{\Gamma^{\prime}, \chi^{\prime}}$ are isospectral if and only if $F_{\Gamma, \chi}(z)=F_{\Gamma^{\prime}, \chi^{\prime}}(z)$, and by Theorem 3.6 this is equivalent to $\vartheta_{\mathcal{L}_{\Gamma, \chi}}(z)=\vartheta_{\mathcal{L}_{\Gamma^{\prime}, \chi^{\prime}}}(z)$ for $M \neq P^{1}(\mathbb{H})$ or to $\vartheta_{D_{2} \cap \mathcal{L}_{\Gamma, \chi}}(z)=$ $\vartheta_{D_{2} \cap \mathcal{L}_{\Gamma^{\prime}, \chi^{\prime}}}(z)$ for $M=P^{1}(\mathbb{H})$. The proof is concluded in light of Definition 4.1.

Remark 4.3. An obvious consequence from the previous characterization is that a twisted Laplace operator cannot be isospectral to an untwisted one. More precisely, $\Delta_{\Gamma, \chi}$ with $\chi$ nontrivial and $\Delta_{\Gamma^{\prime}, 1_{\Gamma^{\prime}}}$ cannot be isospectral since the weight 0 is in $\mathcal{L}_{\Gamma^{\prime}, 1_{\Gamma^{\prime}}}$ but not in $\mathcal{L}_{\Gamma, \chi}$.

The next goal is to show explicit examples of isospectral twisted and untwisted Laplace operators. We will restrict our attention to the case when $\Gamma$ is a cyclic subgroup of $G$ as in \$3.4. By Theorem 4.2, following the notation in (3.20), it is sufficient, for fixed $q$ and $n$, to 
TABLE 1. Twisted $\|\cdot\|_{1}$-isospectral families among $\mathcal{L}_{q, s, u}$ for $2 \leq m \leq 4$ and $q \leq 7$

\begin{tabular}{|c|c|c|c|c|c|c|c|c|c|c|c|c|c|c|}
\hline$q$ & $s$ & $u$ & $q$ & $s$ & $u$ & $q$ & $s$ & $u$ & $q$ & $s$ & $u$ & $q$ & $s$ & $u$ \\
\hline 4 & {$[0,1]$} & 1 & 4 & {$[0,1,1]$} & 1 & 4 & {$[0,0,0,1]$} & 1 & 5 & {$[0,0,0,1]$} & 1 & 7 & {$[0,0,1,2]$} & 1 \\
\hline 4 & {$[1,2]$} & 1 & 4 & {$[1,1,2]$} & 1 & 4 & {$[0,0,1,2]$} & 1 & 5 & {$[0,0,1,2]$} & 1 & 7 & {$[0,0,1,2]$} & 2 \\
\hline 5 & {$[0,1]$} & 1 & 5 & {$[0,0,1]$} & 1 & 4 & {$[0,1,2,2]$} & 1 & 5 & {$[0,0,1,2]$} & 2 & 7 & {$[0,1,2,3]$} & 1 \\
\hline 5 & {$[1,2]$} & 1 & 5 & {$[0,1,2]$} & 1 & 4 & {$[1,2,2,2]$} & 1 & 5 & {$[0,1,1,2]$} & 1 & 7 & {$[0,1,2,3]$} & 2 \\
\hline 5 & {$[1,2]$} & 2 & 5 & {$[0,1,2]$} & 2 & 4 & {$[0,0,1,1]$} & 1 & 5 & {$[1,1,2,2]$} & 1 & 7 & {$[0,1,2,3]$} & 3 \\
\hline 7 & {$[1,2]$} & 1 & 7 & {$[0,1,2]$} & 1 & 4 & {$[0,1,1,2]$} & 1 & 5 & {$[1,1,2,2]$} & 2 & & & \\
\hline 7 & {$[1,2]$} & 2 & 7 & {$[0,1,2]$} & 2 & 4 & {$[1,1,2,2]$} & 1 & 7 & {$[1,1,2,2]$} & 1 & & & \\
\hline 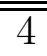 & $\overline{\overline{0,0,1]}}$ & 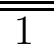 & 7 & {$[1,2,3]$} & 1 & 4 & {$[0,1,1,1]$} & 1 & 7 & {$[1,1,2,3]$} & 1 & & & \\
\hline 4 & $0,1,2]$ & 1 & 7 & {$[1,2,3]$} & 2 & 4 & {$[1,1,1,2]$} & 1 & & & & & & \\
\hline 4 & $1,2,2]$ & 1 & 7 & {$[1,2,3]$} & 3 & & & & & & & & & \\
\hline
\end{tabular}

TABLE 2. Untwisted $\|\cdot\|_{1}$-isospectral families among $\mathcal{L}_{q, s}$ for $2 \leq m \leq 5$ and $q \leq 15$

\begin{tabular}{|c|c|c|c|c|c|c|c|c|c|}
\hline$q$ & $s$ & $q$ & $s$ & $q$ & $s$ & $q$ & $s$ & $q$ & $s$ \\
\hline 11 & {$[1,2,3]$} & 11 & {$[0,1,2,3]$} & 15 & {$[0,1,2,6]$} & 13 & {$[0,0,1,2,5]$} & 15 & {$[0,1,2,5,6]$} \\
\hline 11 & {$[1,2,4]$} & 11 & {$[0,1,2,4]$} & 15 & {$[0,1,3,4]$} & 13 & {$[0,0,1,3,4]$} & 15 & {$[0,1,3,4,5]$} \\
\hline 13 & {$[1,2,3]$} & 13 & {$[0,1,2,3]$} & 15 & {$[1,2,5,6]$} & 13 & {$[0,1,2,3,4]$} & 15 & {$[1,2,5,5,6]$} \\
\hline 13 & {$[1,2,4]$} & 13 & {$[0,1,2,4]$} & 15 & {$[1,3,4,5]$} & 13 & {$[0,1,2,3,5]$} & 15 & {$[1,3,4,5,5]$} \\
\hline 13 & {$[1,2,5]$} & 13 & {$[0,1,2,5]$} & 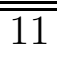 & $\overline{[\overline{[0,0,1,2,3]}}$ & 15 & {$[0,0,1,2,6]$} & 15 & {$[1,2,3,6,6]$} \\
\hline 13 & {$[1,3,4]$} & 13 & {$[0,1,3,4]$} & 11 & {$[0,0,1,2,4]$} & 15 & {$[0,0,1,3,4]$} & 15 & {$[1,3,3,4,6]$} \\
\hline 15 & {$[1,2,6]$} & 13 & {$[1,2,3,4]$} & 13 & {$[0,0,1,2,3]$} & & & & \\
\hline 15 & {$[1,3,4]$} & 13 & {$[1,2,3,5]$} & 13 & {$[0,0,1,2,4]$} & & & & \\
\hline
\end{tabular}

check whether $\mathcal{L}_{q, s, u}$ and $\mathcal{L}_{q, s^{\prime}, u^{\prime}}\left(\right.$ or $\mathcal{L}_{q, s, u}^{*}$ and $\left.\mathcal{L}_{q, s^{\prime}, u^{\prime}}^{*}\right)$ are $\|\cdot\|$-isospectral, that is, $N_{\mathcal{L}_{q, s, u}}(k)=$ $N_{\mathcal{L}_{q, s^{\prime}, u^{\prime}}}(k)$ for any $k \geq 0$.

By using the computer program Sage [Sage, for low values of $n$ and $q$, we found all pairs $(s, u),\left(s^{\prime}, u^{\prime}\right)$ such that $\mathcal{L}_{q, s, u}$ and $\mathcal{L}_{q, s^{\prime}, u^{\prime}}\left(\right.$ and also $\mathcal{L}_{q, s, u}^{*}$ and $\left.\mathcal{L}_{q, s^{\prime}, u^{\prime}}^{*}\right)$ are $\|\cdot\|$-isospectral. Clearly, $\mathcal{L}_{q, s, u}$ and $\mathcal{L}_{q, s, q-u}\left(\right.$ and also $\mathcal{L}_{q, s, u}^{*}$ and $\left.\mathcal{L}_{q, s, q-u}^{*}\right)$ are trivially $\|\cdot\|$-isospectral, thus we assume $0 \leq u \leq q / 2$.

4.2. Isospectral examples covered by spheres. Table 1 shows families of affine lattices of the form $\mathcal{L}_{q, s, u}$ that are mutually $\|\cdot\|_{1}$-isospectral, for $2 \leq n \leq 4, q \leq 7$ and $1 \leq u \leq\left\lfloor\frac{q}{2}\right\rfloor$. They induce families of twisted isospectral non-isometric orbifolds covered by $S^{2 n}$ and $S^{2 n-1}$. Table 2 shows all families of lattices of the form $\mathcal{L}_{q, s}$ that are mutually $\|\cdot\|_{1}$-isospectral, for $2 \leq n \leq 5$ and $q \leq 15$. They induce families of untwisted isospectral non-isometric orbifolds covered by $S^{2 n}$ and $S^{2 n-1}$. The cases (assuming $q>2$ ) that an orbifold covered by a sphere with cyclic fundamental group is a manifold is when $n$ is odd and $\operatorname{gcd}\left(s_{j}, q\right)=1$ for all $1 \leq j \leq n$.

Here are some remarks and particular examples.

Remark 4.4. Ikeda [Ik80a] showed a simple characterization and several examples of untwisted isospectral lens spaces by using (3.22). His method works for $q$ prime, but it can be extended in general. The article GM06 contains a numerical study of isospectral lens spaces based on Ikeda's method, though their results are correct for $q$ prime but not in general.

Remark 4.5. Shams [Sh11] extended Ikeda's method to orbifold lens spaces, that is, spaces of the form $\Gamma_{q, s} \backslash S^{2 n-1}$ with $\operatorname{gcd}\left(q, s_{j}\right) \neq 1$ for some $1 \leq j \leq n$. He obtained pairs of isospectral non-isometric orbifold lens spaces in any dimension greater than or equal to 9 . The pair 
$\mathcal{L}_{15 ;(1,2,6)}, \mathcal{L}_{15 ;(1,3,4)}$ in Table 2 gives an example in dimension 5 and 6 , and the same table contains several examples in dimensions 7 and 8. The author believes that such examples do not exist in any dimension smaller than 5, even in the case of non-cyclic fundamental group.

Conjecture 4.6. There are no pairs of untwisted isospectral non-isometric orbifolds covered by $S^{n}$ with $n \leq 4$.

Remark 4.7. In support to the previous conjecture, Ikeda and Yamamoto [IY79] [Ya80] have already proved that there are no untwisted isospectral non-isometric 3-dimensional lens spaces. In other words, they proved the conjecture for manifolds covered by $S^{3}$. Our computations did not find any untwisted isospectral non-isometric 3 or 4-dimensional orbifold lens spaces with $q \leq 200$. However, Table 1 shows twisted isospectral 3 and 4-dimensional orbifold lens spaces.

Example 4.8. Here we give a simple proof of $\|\cdot\|_{1}$-isospectrality for the first example in Table 1 , namely $\mathcal{L}:=\mathcal{L}_{4,(0,1), 1}$ and $\mathcal{L}^{\prime}:=\mathcal{L}_{4,(1,2), 1}$. We have that

$$
\mathcal{L}=\left\{(a, b) \in \mathbb{Z}^{2}: b \equiv 1 \quad(\bmod 4)\right\}, \quad \mathcal{L}^{\prime}=\left\{(a, b) \in \mathbb{Z}^{2}: a+2 b \equiv 1 \quad(\bmod 4)\right\} .
$$

Figure 1 shows a picture of them. Let $\varphi: \mathcal{L}^{\prime} \rightarrow \mathbb{Z}^{2}$ given by

$$
\varphi(a, b)=\left\{\begin{array}{lll}
(a, b) & \text { if } b \equiv 1 \quad(\bmod 4) \\
(-a,-b) & \text { if } b \equiv 3 \quad(\bmod 4) \\
(b, a) & \text { if } b \equiv 0 \quad(\bmod 2)
\end{array}\right.
$$

Clearly, $\varphi$ is $\|\cdot\|_{1}$-preserving and one can easily check that it is injective and $\varphi\left(\mathcal{L}^{\prime}\right)=\mathcal{L}$. It follows immediately that $\mathcal{L}$ and $\mathcal{L}^{\prime}$ are $\|\cdot\|_{1}$-isospectral.

Remark 4.9. Dryden, Gordon, Greenwald and Webb DGGW08 proved that a manifold cannot be untwisted isospectral to an orbifold (that is not a manifold) when they have a common Riemannian cover. The second family in Table 1 shows that this is not true for twisted isospectrality. Indeed, the pair $\mathcal{L}_{5,(0,1), 1}, \mathcal{L}_{5,(1,2), 1}$ induces the twisted isospectral pair $\left(\Gamma_{5,(0,1)} \backslash S^{3}, \chi_{1}\right),\left(\Gamma_{5,(1,2)} \backslash S^{3}, \chi_{1}\right)$, where $\Gamma_{5,(1,2)} \backslash S^{3}$ is a manifold and $\Gamma_{5,(0,1)} \backslash S^{3}$ is an orbifold.

Example 4.10. Here we give a proof of $\|\cdot\|_{1}$-isospectrality of $\mathcal{L}_{q,(1,2), 1}$ and $\mathcal{L}_{q,(1,2), 2}$ for every $q$ odd. Such as in Example 4.8, we will give a $\|\cdot\|_{1}$-preserving bijection between them.

Let $\mathcal{L}=\mathcal{L}_{q,(1,2)}=\left\{(a, b) \in \mathbb{Z}^{2}: a+2 b \equiv 0(\bmod q)\right\}$ (see Figure 2 for $q=7$ ), thus $\mathcal{L}_{q,(1,2), 1}=\mathcal{L}+(1,0)$ and $\mathcal{L}_{q,(1,2), 2}=\mathcal{L}+(0,1)$. A nice way to see the points in these affine lattices is to see the points in $\mathcal{L}$ moving the origin to $(-1,0)$ and $(0,-1)$ respectively. Hence, we are looking for a bijection $\varphi: \mathcal{L} \rightarrow \mathcal{L}$ such that $\|\varphi(\mu)-(0,-1)\|_{1}=\|\mu-(-1,0)\|_{1}$ for every $\mu \in \mathcal{L}$. One can check that this condition is satisfied by the function

$$
\varphi(a, b)= \begin{cases}(a, b) & \text { if } a, b \geq 1, \text { or } a, b \leq-1, \\ (-a,-b) & \text { if }-a, b \geq 1, \text { or }-a, b \leq-1, \\ (0, a) & \text { if } a \leq-1 \text { and } b=0\end{cases}
$$

One can easily check that $\mathcal{L}_{q,\left(0^{m}, 1,2,\right), 1}$ and $\mathcal{L}_{q,\left(0^{m}, 1,2\right), 2}$ are also $\|\cdot\|_{1}$-isospectral for every $q$ odd. Here $0^{m}$ in $s$ and $s^{\prime}$ means a zero repeated $m$ times.

Remark 4.11. One can check that, for a fixed isospectral pair $(q, s, u)$ and $\left(q, s^{\prime}, u^{\prime}\right)$, if we add zero coordinates to $s$ and $s^{\prime}$, then we again obtain isospectrality. This fact was already known (see [Sh11, Cor. 3.2.4]). 


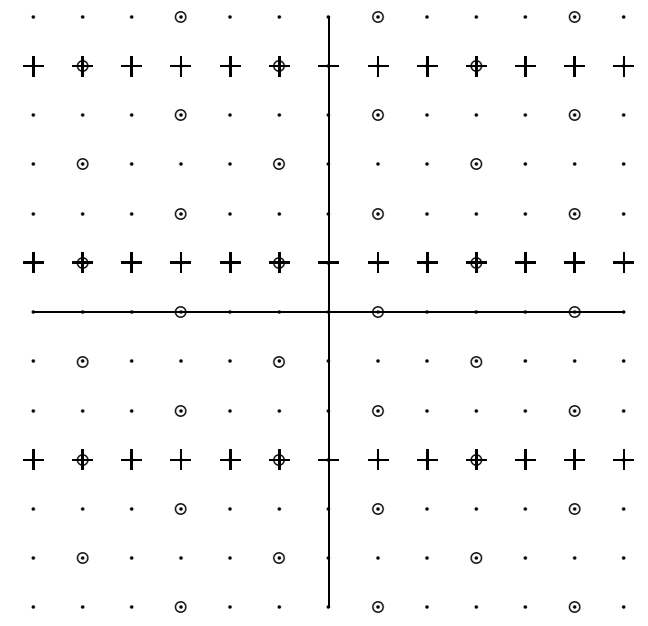

FigURE 1. $\mathcal{L}_{4,(0,1), 1}, \mathcal{L}_{4,(1,2), 1}$

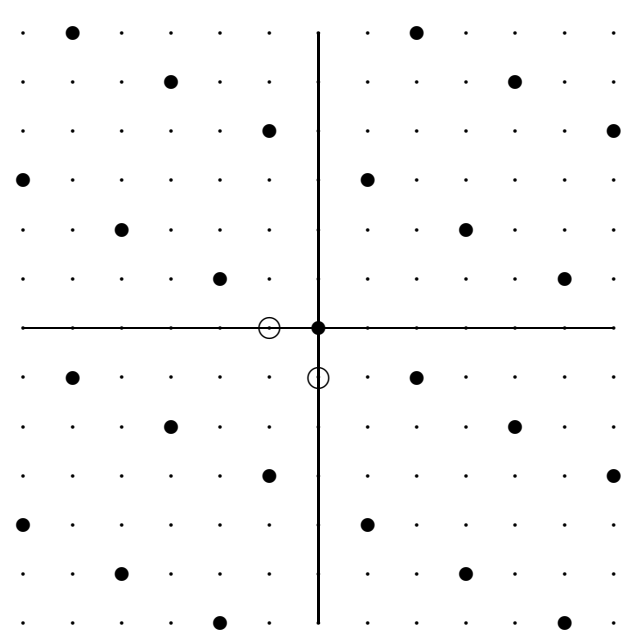

FIGURE $2 . \mathcal{L}_{7,(1,2)}$

TABLE 3 . Twisted $\|\cdot\|_{1}$-isospectral families among $\mathcal{L}_{q, s, u}^{*}$ for $2 \leq n \leq 3$ and $q \leq 6$

\begin{tabular}{ccc}
$q$ & $s$ & $u$ \\
\hline 4 & {$[0,1,3]$} & 1 \\
4 & {$[1,1,2]$} & 1 \\
\hline 6 & {$[0,1,5]$} & 1 \\
6 & {$[1,2,3]$} & 1 \\
\hline 6 & {$[0,1,5]$} & 2 \\
6 & {$[1,2,3]$} & 2 \\
\hline 6 & {$[0,1,5]$} & 3 \\
6 & {$[1,2,3]$} & 3 \\
\hline
\end{tabular}

\begin{tabular}{ccc}
$q$ & $s$ & $u$ \\
\hline 6 & {$[1,1,4]$} & 1 \\
6 & {$[1,1,4]$} & 2 \\
\hline \hline 4 & {$[0,0,1,3]$} & 1 \\
4 & {$[0,1,1,2]$} & 1 \\
4 & {$[1,2,2,3]$} & 1 \\
\hline 4 & {$[0,0,1,3]$} & 2 \\
4 & {$[0,1,1,2]$} & 2 \\
4 & {$[1,2,2,3]$} & 2 \\
\hline
\end{tabular}

\begin{tabular}{ccc}
$q$ & $s$ & $u$ \\
\hline 4 & {$[1,1,1,1]$} & 1 \\
4 & {$[1,1,1,1]$} & 2 \\
4 & {$[1,1,3,3]$} & 1 \\
\hline 5 & {$[1,2,3,4]$} & 1 \\
5 & {$[1,2,3,4]$} & 2 \\
\hline 6 & {$[0,0,1,5]$} & 1 \\
6 & {$[2,3,3,4]$} & 1 \\
\hline 6 & {$[0,0,1,5]$} & 2 \\
6 & {$[2,3,3,4]$} & 2 \\
\hline
\end{tabular}

\begin{tabular}{ccc}
$q$ & $s$ & $u$ \\
\hline 6 & {$[0,0,1,5]$} & 3 \\
6 & {$[2,3,3,4]$} & 3 \\
\hline 6 & {$[1,1,1,3]$} & 1 \\
6 & {$[1,1,1,3]$} & 3 \\
6 & {$[1,1,5,5]$} & 1 \\
6 & {$[1,1,5,5]$} & 3 \\
6 & {$[1,3,3,5]$} & 1 \\
6 & {$[1,3,3,5]$} & 3 \\
\hline
\end{tabular}

TABLE 4 . Untwisted $\|\cdot\|_{1}$-isospectral families among $\mathcal{L}_{q, s}^{*}$ for $2 \leq n \leq 3$ and $q \leq 10$

\begin{tabular}{cc}
$q$ & $s$ \\
\hline 6 & {$[0,1,5]$} \\
6 & {$[1,2,3]$} \\
\hline 9 & {$[0,1,8]$} \\
9 & {$[1,2,6]$} \\
\hline \hline
\end{tabular}

\begin{tabular}{cc}
$q$ & $s$ \\
\hline 4 & {$[0,0,1,3]$} \\
4 & {$[0,1,1,2]$} \\
4 & {$[1,2,2,3]$} \\
\hline 6 & {$[0,0,1,5]$} \\
6 & {$[2,3,3,4]$} \\
\hline
\end{tabular}

\begin{tabular}{cc}
$q$ & $s$ \\
\hline 7 & {$[0,1,2,4]$} \\
7 & {$[1,2,5,6]$} \\
\hline 8 & {$[0,0,1,7]$} \\
8 & {$[1,2,2,3]$} \\
8 & {$[1,4,4,7]$} \\
\hline
\end{tabular}

\begin{tabular}{cc}
$q$ & $s$ \\
\hline 8 & {$[0,1,1,6]$} \\
8 & {$[1,1,2,4]$} \\
\hline 8 & {$[1,1,3,3]$} \\
8 & {$[1,1,7,7]$} \\
\hline
\end{tabular}

\begin{tabular}{cc}
$q$ & $s$ \\
\hline 10 & {$[0,0,1,9]$} \\
10 & {$[2,5,5,8]$} \\
\hline 10 & {$[0,1,1,8]$} \\
10 & {$[1,2,2,5]$} \\
\hline
\end{tabular}

4.3. Isospectral examples covered by complex projective spaces. Table 3 shows all families of affine lattices of the form $\mathcal{L}_{q, s, u}^{*}$ that are mutually $\|\cdot\|_{1}$-isospectral, for $2 \leq n \leq 3$, $q \leq 6$ and $1 \leq u \leq\left\lfloor\frac{q}{2}\right\rfloor$. They induce families of $2 n$-dimensional twisted isospectral nonisometric orbifolds covered by $P^{n}(\mathbb{C})$. Table 4 shows all families of lattices of the form $\mathcal{L}_{q, s}^{*}$ that are mutually $\|\cdot\|_{1}$-isospectral, for $2 \leq n \leq 3$ and $q \leq 10$. They induce families of $2 n$-dimensional untwisted isospectral non-isometric orbifolds covered by $P^{n}(\mathbb{C})$. Every orbifold in this case is not a manifold. 
TABLE 5. Twisted $\|\cdot\|_{\infty}$-isospectral families among $\mathcal{L}_{q, s, u}$ for $n=2$ and $q \leq 9$

\begin{tabular}{ccc}
$q$ & $s$ & $u$ \\
\hline 4 & {$[0,1]$} & 1 \\
4 & {$[1,2]$} & 1 \\
\hline 4 & {$[0,1]$} & 2 \\
4 & {$[1,2]$} & 2 \\
\hline 5 & {$[1,1]$} & 2 \\
5 & {$[1,2]$} & 1 \\
5 & {$[1,2]$} & 2 \\
\hline 6 & {$[0,1]$} & 1 \\
6 & {$[2,3]$} & 1 \\
\hline
\end{tabular}

\begin{tabular}{ccc}
$q$ & $s$ & $u$ \\
\hline 6 & {$[0,1]$} & 2 \\
6 & {$[2,3]$} & 2 \\
\hline 6 & {$[0,1]$} & 3 \\
6 & {$[2,3]$} & 3 \\
\hline 6 & {$[1,1]$} & 1 \\
6 & {$[1,1]$} & 3 \\
6 & {$[1,3]$} & 1 \\
6 & {$[1,3]$} & 3 \\
\hline
\end{tabular}

\begin{tabular}{ccc}
$q$ & $s$ & $u$ \\
\hline 7 & {$[1,2]$} & 1 \\
7 & {$[1,2]$} & 3 \\
\hline 8 & {$[0,1]$} & 1 \\
8 & {$[1,4]$} & 3 \\
\hline 8 & {$[0,1]$} & 2 \\
8 & {$[1,2]$} & 2 \\
8 & {$[1,4]$} & 2 \\
\hline 8 & {$[0,1]$} & 3 \\
8 & {$[1,4]$} & 1 \\
\hline
\end{tabular}

\begin{tabular}{ccc}
$q$ & $s$ & $u$ \\
\hline 8 & {$[0,1]$} & 4 \\
8 & {$[1,4]$} & 4 \\
\hline 8 & {$[1,1]$} & 1 \\
8 & {$[1,1]$} & 3 \\
8 & {$[1,3]$} & 1 \\
8 & {$[1,3]$} & 3 \\
\hline 8 & {$[1,1]$} & 2 \\
8 & {$[1,3]$} & 2 \\
\hline
\end{tabular}

\begin{tabular}{ccc}
$q$ & $s$ & $u$ \\
\hline 8 & {$[1,2]$} & 1 \\
8 & {$[1,2]$} & 3 \\
\hline 9 & {$[1,1]$} & 4 \\
9 & {$[1,2]$} & 4 \\
\hline 9 & {$[1,3]$} & 2 \\
9 & {$[1,3]$} & 4 \\
\hline
\end{tabular}

TABLE 6. Untwisted $\|\cdot\|_{\infty}$-isospectral families among $\mathcal{L}_{q, s}$ for $n=2$ and $q \leq 20$

\begin{tabular}{|c|c|c|c|c|c|c|c|c|c|c|c|c|c|}
\hline$q$ & $s$ & $q$ & $s$ & $q$ & $s$ & $q$ & $s$ & $q$ & $s$ & $q$ & $s$ & $q$ & $s$ \\
\hline 4 & $\overline{[0,1]}$ & 8 & {$[0,1]$} & 12 & $0,1]$ & 12 & {$[2,3]$} & 14 & {$[1,2]$} & 16 & {$[1,2]$} & 18 & {$[1,2]$} \\
\hline 4 & {$[1,2]$} & 8 & {$[1,4]$} & 12 & $1,6]$ & 12 & {$[3,4]$} & 14 & {$[1,4]$} & 16 & {$[1,6]$} & 18 & {$[1,4]$} \\
\hline 6 & {$[0,1]$} & 10 & {$[0,1]$} & 12 & $1,2]$ & 14 & {$[0,1]$} & 16 & {$[\overline{[0,1]}$} & 18 & {$[0,1]$} & 18 & {$[1,6]$} \\
\hline 6 & {$[2,3]$} & 10 & {$[2,5]$} & 12 & $1,4]$ & 14 & {$[2,7]$} & 16 & {$[1,8]$} & 18 & {$[2,9]$} & 18 & {$[2,3]$} \\
\hline
\end{tabular}

Remark 4.12. We explain here the first example in Table 4 and the second, third and fourth example in Table 3, Let $q=6, s=(0,1,5)$ and $s^{\prime}=(1,2,3)$. One can check that

$$
\begin{aligned}
& \mathcal{L}:=\mathcal{L}_{q, s}^{*}=\mathbb{Z}(6,0,-6) \oplus \mathbb{Z}(-2,1,1)=\left\{(a, b, c) \in A_{2}: b \equiv c \quad(\bmod 6)\right\}, \\
& \mathcal{L}^{\prime}:=\mathcal{L}_{q, s^{\prime}}^{*}=\mathbb{Z}(0,6,-6) \oplus \mathbb{Z}(1,-2,1)=\left\{(a, b, c) \in A_{2}:-b \equiv 2 c \quad(\bmod 6)\right\} .
\end{aligned}
$$

It follows immediately that $\mathcal{L}_{q, s, u}^{*}$ and $\mathcal{L}_{q, s^{\prime}, u}^{*}$ are $\|\cdot\|_{1}$-isospectral for any $u$ since $\mathcal{L}$ and $\mathcal{L}^{\prime}$ are related by the permutation $(a, b, c) \mapsto(b, a, c)$ which clearly preserves $\|\cdot\|_{1}$. On the other hand, the orbifolds $\Gamma_{q, s} \backslash P^{2}(\mathbb{C})$ and $\Gamma_{q, s^{\prime}} \backslash P^{2}(\mathbb{C})$ have dimension 4 and are non-isometric by Proposition 2.8 since every coefficient in $s^{\prime}$ is not divisible by $q$.

Remark 4.13. The minimum dimension of twisted or untwisted orbifold covered by complex projective spaces is 4 , thus $n=2$. Indeed, if $n=1$ then $\mathcal{L}_{q, s}=\mathbb{Z}(q,-q)$ for any $s \in \mathbb{Z}$ coprime to $q$, thus there is only one such orbifold whose fundamental group order is $q$, and the assertion follows since $q$ is a spectral invariant (see Remark 3.11).

4.4. Isospectral examples covered by the quaternion projective line. Table 5 shows all families of affine lattices of the form $\mathcal{L}_{q, s, u}$ that are mutually $\|\cdot\|_{\infty}$-isospectral, for $n=2$, $q \leq 9$ and $1 \leq u \leq\left\lfloor\frac{q}{2}\right\rfloor$. They induce families of 4-dimensional twisted isospectral orbifolds covered by $P^{1}(\mathbb{H})$. Table 6 shows all families of lattices of the form $\mathcal{L}_{q, s}$ that are mutually $\|\cdot\|_{\infty^{-}}$ isospectral, for $n=2$ and $q \leq 10$. They induce families of 4-dimensional untwisted isospectral non-isometric orbifolds covered by $P^{1}(\mathbb{H})$. Similarly as in the case of $P^{n}(\mathbb{C})$, every orbifold in this subsection is not a manifold.

Example 4.14. We study here the first pair in Table 6 and the two first pairs in Table 5. Let $q=4, s=(0,1), s^{\prime}=(1,2)$. The corresponding congruence lattices $\mathcal{L}:=\mathcal{L}_{q, s}$ and $\mathcal{L}^{\prime}=\mathcal{L}_{q, s^{\prime}}$ are the same as in Example 4.8, One can easily check that

$$
D_{2} \cap \mathcal{L}=\mathbb{Z}(0,4) \oplus \mathbb{Z}(2,0) \quad \text { and } \quad D_{2} \cap \mathcal{L}^{\prime}=\mathbb{Z}(4,0) \oplus \mathbb{Z}(0,2) .
$$


It follows immediately that $D_{2} \cap \mathcal{L}$ and $D_{2} \cap \mathcal{L}^{\prime}$ are $\|\cdot\|_{\infty}$-isospectral since they differ only by a permutation of coordinates, which preserves $\|\cdot\|_{\infty}$. However, the orbifolds $\Gamma_{q, s} \backslash P^{1}(\mathbb{H})$ and $\Gamma_{q, s^{\prime}} \backslash P^{1}(\mathbb{H})$ are not isometric by Proposition 2.9.

Remark 4.15. Every example in Table 6 is explained as in the previous example, that is, $D_{2} \cap \mathcal{L}_{q, s}$ and $D_{2} \cap \mathcal{L}_{q, s^{\prime}}$ are $\|\cdot\|_{\infty}$-isometric.

4.5. More remarks. Here we include more remarks. For a recent account on spectral theory of orbifolds we refer the reader to Go12.

Remark 4.16. Miatello and Rossetti MR02 studied twisted isospectrality on compact flat manifolds. It is not difficult to construct a twisted isospectral pair between a compact flat manifold and a compact flat orbifold with singularities like in Remark 4.9, Gordon and Rossetti GR03 gave similar examples replacing twisted spectrum by the middle spectrum.

Remark 4.17. Rossetti, Schueth and Weilandt [RSW08] showed examples of untwisted isospectral orbifolds such that the maximal isotropy order of singular points are different (see also [SSW06]). Such examples do not appear in this article. Indeed, for $M=G / K=P^{n}(\mathbb{C}), P^{1}(\mathbb{H})$, it is clear that the maximal isotropy order of $\Gamma_{q, s} \backslash M$ is $q$ since $\Gamma_{q, s} \subset g K g^{-1}$ for some $g \in G$ (see [RSW08, Thm. 2.5 (ii)]). When $M=S^{2 n}, S^{2 n-1}$, the maximal isotropy order $\Gamma_{q, s} \backslash M$ is determined by the set $\left\{\operatorname{gcd}\left(s_{i}, q\right): 1 \leq i \leq n\right\}$. One can check that these sets coincide for each untwisted isospectral pair in Table 2 (cf. [FPS14, §3.4]).

4.6. A non-cyclic example. We set $\Gamma=\left\langle\gamma_{1}, \gamma_{2}, \gamma_{3}\right\rangle$ and $\Gamma^{\prime}=\left\langle\gamma_{1}^{\prime}, \gamma_{2}^{\prime}, \gamma_{3}^{\prime}\right\rangle$, where

$$
\begin{aligned}
& \gamma_{1}=\operatorname{diag}\left(-I_{2},-I_{2}, \quad I_{2}, \quad I_{2}, \quad I_{2}, \quad I_{2}\right), \quad \gamma_{1}^{\prime}=\operatorname{diag}\left(-I_{2},-I_{2}, \quad I_{2}, \quad I_{2}, \quad I_{2}, I_{2}\right), \\
& \gamma_{2}=\operatorname{diag}\left(I_{2}, \quad I_{2},-I_{2},-I_{2}, \quad I_{2}, I_{2}\right), \quad \gamma_{2}^{\prime}=\operatorname{diag}\left(-I_{2}, I_{2},-I_{2}, I_{2}, I_{2}, I_{2}\right) \text {, } \\
& \gamma_{3}=\operatorname{diag}\left(I_{2}, \quad I_{2}, I_{2}, I_{2},-I_{2},-I_{2}\right), \quad \gamma_{3}^{\prime}=\operatorname{diag}\left(-I_{2},-I_{2},-I_{2},-I_{2},-I_{2},-I_{2}\right) \text {. }
\end{aligned}
$$

Here $I_{2}$ denotes the $2 \times 2$ identity matrix. Then $\Gamma$ and $\Gamma^{\prime}$ are subgroups of the maximal torus in $\mathrm{SO}(12)$ and the orbifolds $\Gamma \backslash S^{11}$ and $\Gamma^{\prime} \backslash S^{11}$ are not manifolds. Indeed, each element in $\Gamma$ and in $\Gamma^{\prime}$ does not act without fixed points. One can check that

$$
\begin{aligned}
& \mathcal{L}_{\Gamma}=\left\{\mu=\left(a_{1}, \ldots, a_{6}\right) \in \mathbb{Z}^{6}: \begin{array}{rr}
a_{1}+a_{2} \equiv 0 & (\bmod 2) \\
a_{3}+a_{4} \equiv 0 & (\bmod 2) \\
a_{5}+a_{6} \equiv 0 & (\bmod 2)
\end{array}\right\} \\
& \mathcal{L}_{\Gamma}^{\prime}=\left\{\mu=\left(a_{1}, \ldots, a_{6}\right) \in \mathbb{Z}^{6}: \begin{array}{rr}
a_{1}+a_{2} \equiv 0 & (\bmod 2) \\
a_{3}+a_{4} \equiv 0 & (\bmod 2) \\
a_{1}+\cdots+a_{6} \equiv 0 & (\bmod 2)
\end{array}\right\} .
\end{aligned}
$$

These lattices were already used by Conway and Sloane in CS92 to give lattices in dimension 6 with the same theta series. In our words, these lattices are two-norm isospectral, it means that for each $t \geq 0$ we have that

$$
\#\left\{\mu \in \mathcal{L}_{\Gamma}:\|\mu\|_{2}=t\right\}=\#\left\{\mu \in \mathcal{L}_{\Gamma^{\prime}}:\|\mu\|_{2}=t\right\}
$$

Here $\|\mu\|_{2}=\sqrt{a_{1}^{2}+\cdots+a_{n}^{2}}$. By [Mi64, this pair induces a pair of 6-dimensional flat tori isospectral with respect to the Laplace operator. Also in [CS92, the authors construct 4dimensional two-norm isospectral lattices. 
Write $\Psi: \mathbb{Z}^{6} \rightarrow(\mathbb{Z} / 2 \mathbb{Z})^{6}$ given by the class modulo 2 in each coordinate. We have that $\mathcal{L}_{\Gamma}=\Psi^{-1}(C)$ and $\mathcal{L}_{\Gamma^{\prime}}=\Psi^{-1}\left(C^{\prime}\right)$, where $C$ and $C^{\prime}$ are the codes in $(\mathbb{Z} / 2 \mathbb{Z})^{6}$ given by

$C: \begin{array}{llllll}0 & 0 & 0 & 0 & 0 & 0 \\ 1 & 1 & 0 & 0 & 0 & 0 \\ 0 & 0 & 1 & 1 & 0 & 0 \\ 0 & 0 & 0 & 0 & 1 & 1 \\ 1 & 1 & 1 & 1 & 1 & 1 \\ 1 & 1 & 1 & 1 & 0 & 0 \\ 1 & 1 & 0 & 0 & 1 & 1 \\ 0 & 0 & 1 & 1 & 1 & 1\end{array}$,

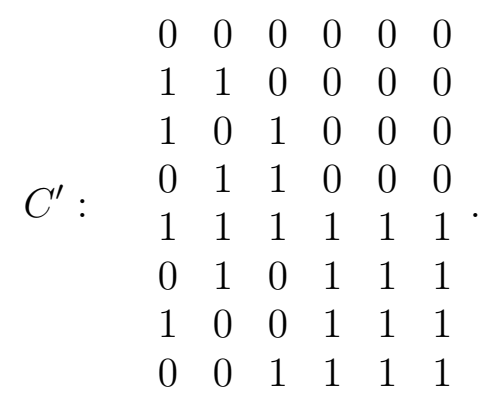

By this clever way to see these lattices, it follows that the lattices are two-norm isospectral since the preimages of each row clearly satisfy (4.2).

It is also clear that the preimages of each row are also one-norm isospectral, then so are $\mathcal{L}_{\Gamma}$ and $\mathcal{L}_{\Gamma^{\prime}}$. By Theorem 4.2 we obtain that the orbifolds $\Gamma \backslash S^{11}$ and $\Gamma^{\prime} \backslash S^{11}$ are isospectral with respect to the untwisted Laplace operator on functions. Moreover, similarly as in Corollary 4.18, this pair induces a pair of isospectral orbifolds covered by $S^{12}$.

Additionally, one can check also immediately that $\mathcal{L}_{\Gamma}$ and $\mathcal{L}_{\Gamma^{\prime}}$ are $\|\cdot\|_{1}^{*}$-isospectral in the sense of [LMR15]. This implies that the orbifolds $\Gamma \backslash S^{11}$ and $\Gamma^{\prime} \backslash S^{11}$ are $p$-isospectral for all $p$ by [LMR15, Thm. 3.9]. However, contrary to all examples in [LMR15], $\Gamma$ and $\Gamma^{\prime}$ are representation equivalent in $\mathrm{SO}(12)$, thus the orbifolds are already strongly equivalent. The fact that $\Gamma$ and $\Gamma^{\prime}$ are almost conjugate (which implies representation equivalent) follows immediately from

$$
\begin{aligned}
& \gamma_{1}=\gamma_{1}^{\prime}, \quad \gamma_{3} \sim \gamma_{1}^{\prime} \gamma_{2}^{\prime}, \quad \gamma_{1} \gamma_{3} \sim \gamma_{1}^{\prime} \gamma_{2}^{\prime} \gamma_{3}^{\prime}, \quad \gamma_{1} \gamma_{2} \gamma_{3}=\gamma_{3}^{\prime}, \\
& \gamma_{2} \sim \gamma_{2}^{\prime}, \quad \gamma_{1} \gamma_{2} \sim \gamma_{2}^{\prime} \gamma_{3}^{\prime}, \quad \gamma_{2} \gamma_{3}=\gamma_{1}^{\prime} \gamma_{3}^{\prime} \text {. }
\end{aligned}
$$

Here, the symbol $\sim$ means that they are conjugate in $\mathrm{SO}(12)$, or equivalently, they have the same eigenvalues.

Any pair of almost conjugate groups given by $n \times n$ diagonal matrices with entries \pm 1 , induces a pair of strongly isospectral orbifolds covered by $S^{2 n-1}$ (and also by $S^{2 n}$ ). Of course, the orbifolds are isometric if and only if the subgroups are conjugate. In [LMR13, §2], there are several such examples. For instance, [LMR13, Example 4.3] shows a family of eight subgroups of $\mathrm{SO}(24)$ that are almost conjugate (and not conjugate) pairwise.

In conclusion, these kind of examples come from Sunada's method (or representation equivalent method) and not exclusively from the one-norm method studied in [LMR15] and in this paper.

4.7. A more general characterization. It is clear that the orbifold covered by $S^{2 n}$ with cyclic fundamental group of order $q$ are in correspondences with the ones covered by $S^{2 n-1}$. This holds because the maximal torus on $\mathrm{SO}(2 n+1)$ and $\mathrm{SO}(2 n)$ coincide. Moreover, we have seen that the characterization of twisted and untwisted isospectral orbifolds with cyclic fundamental group is the same in both cases since the associated congruence lattices coincide. We conclude this article by giving a more general relation between isospectral orbifolds covered by $S^{2 n}$ and $S^{2 n-1}$.

Corollary 4.18. Let $G=\mathrm{SO}(2 n+1), H=\left\{g \in G: g e_{2 n+1}=e_{2 n+1}\right\} \simeq \operatorname{SO}(2 n)$ and $K=\left\{g \in G: g e_{2 n+1}=e_{2 n+1}, g e_{2 n}=e_{2 n}\right\} \simeq \mathrm{SO}(2 n-1)$. Let $\Gamma$ and $\Gamma^{\prime}$ be finite subgroups of $H$ and let $\chi$ and $\chi^{\prime}$ be representations of $\Gamma$ and $\Gamma^{\prime}$ respectively. Then, $\Delta_{S^{2 n}, \Gamma, \chi}$ and $\Delta_{S^{2 n}, \Gamma^{\prime}, \chi^{\prime}}$ are isospectral if and only if $\Delta_{S^{2 n-1}, \Gamma, \chi}$ and $\Delta_{S^{2 n-1}, \Gamma^{\prime}, \chi^{\prime}}$ are isospectral 
Proof. Throughout the proof, $\pi_{k}$ and $\sigma_{k}$ denote the elements in $\widehat{G}_{H}$ and $\widehat{H}_{K}$ respectively with highest weight $k \varepsilon_{1}$. By the classical branching law from $G$ to $H$ (see for instance $\mathrm{Kn}$, Thm. 9.16]), we have that $\left.\pi_{k}\right|_{H} \simeq \bigoplus_{l=0}^{k} \sigma_{l}$. Consequently,

$$
\begin{aligned}
{\left[\chi:\left.\pi_{k}\right|_{\Gamma}\right] } & =\sum_{l=0}^{k}\left[\chi:\left.\sigma_{l}\right|_{\Gamma}\right], \\
{\left[\chi^{\prime}:\left.\pi_{k}\right|_{\Gamma^{\prime}}\right] } & =\sum_{l=0}^{k}\left[\chi^{\prime}:\left.\sigma_{l}\right|_{\Gamma^{\prime}}\right],
\end{aligned}
$$

for every $k \geq 0$. It follows that $\left[\chi:\left.\pi_{k}\right|_{\Gamma}\right]=\left[\chi^{\prime}:\left.\pi_{k}\right|_{\Gamma^{\prime}}\right]$ for every $k \geq 0$ if and only if $\left[\chi^{\prime}:\left.\sigma_{k}\right|_{\Gamma}\right]=\left[\chi^{\prime}:\left.\sigma_{k}\right|_{\Gamma^{\prime}}\right]$ for every $k \geq 0$, hence the proof is complete, by Theorem 3.1 .

This implies that each pair of $(2 n-1)$-dimensional untwisted isospectral spherical space forms (or orbifolds) in [Ik80a], [Ik83], Gi85, Wo01, GM06], Sh11, [LMR15, [DD14, induces a pair of $2 n$-dimensional isospectral orbifolds covered by $S^{2 n}$.

Remark 4.19. There is a similar result in [Pe96, page 261] for $\chi$ trivial. Here, Pesce describes a method to produce a pair of isospectral orbifolds covered by $P^{2 n-1}(\mathbb{C})$ from a pair of isospectral manifolds covered by $S^{2 n-1}$, making use of [Pe96, Lemme 9].

\section{REFERENCES}

[BF08] Bauer W., Furutani K. Zeta regularized determinant of the Laplacian for classes of spherical space forms. J. Geom. Phys. 58:1 (2008), 64-88. DOI: 10.1016/j.geomphys.2007.09.007.

[Bä96] Bär C. The Dirac operator on space forms of positive curvature. J. Math Soc. Japan 48:1 (1996), 69-83. DOI: 10.2969/jmsj/04810069.

[BR] Beck M., RoBins S. Computing the continuous discretely. Integer-point enumeration in polyhedra. Undergrad. Texts Math., Springer New York, 2007. DOI: 10.1007/978-0-387-46112-0.

[Bo15] Boldt S. Properties of the Dirac spectrum on three dimensional lens spaces. arXiv:1504.03121 (2015).

[BL14] Boldt S., Lauret E.A. An explicit formula for the Dirac multiplicities on lens spaces. arXiv:1412.2599 (2014).

[CT04] Cagliero L., Tirao P. A closed formula for weight multiplicities of representations of $\operatorname{Sp}_{2}(\mathbb{C})$. Manuscripta Math. 115:4 (2004), 417-426. DOI: 10.1007/s00229-004-0499-0

[CB94a] Carletti E., Monti Bragadin G. On Dirichlet series associated with polynomials. Proc. Amer. Math. Soc. 121:1 (1994), 33-37. DOI: 10.1090/S0002-9939-1994-1179586-5.

[CB94b] Carletti E., Monti Bragadin G. On Minakshisundaram-Pleijel zeta functions of spheres. Proc. Amer. Math. Soc. 122:4 (1994), 993-1001. DOI: 10.1090/S0002-9939-1994-1249872-9.

[CS92] Conway J.H., Sloane N.J.A. Four-dimensional lattices with the same theta series. Int. Math. Res. Not. IMRN 1992:4 (1992), 93-96. DOI: 10.1155/S1073792892000102.

[DD14] DeFord D., Doyle P. Cyclic groups with the same Hodge series. arXiv:1404.2574 (2014).

[DGGW08] Dryden E., Gordon C., Greenwald S., Webb D. Asymptotic expansion of the heat kernel for orbifolds. Michigan Math. J. 56:1 (2008), 205-238. DOI: 10.1307/mmj/1213972406.

[FPS14] Farsi C., Proctor E., Seaton C. Г-extensions of the spectrum of an orbifold. Trans. Amer. Math. Soc. 366:7 (2014), 3881-3905. DOI: 10.1090/S0002-9947-2013-06082-5.

[FH] Fulton W., Harris J. Representation Theory, A first course. Springer-Verlag New York, 2004. DOI: 10.1007/978-1-4612-0979-9.

[Gi85] GILKEY P. On spherical space forms with meta-cyclic fundamental group which are isospectral but not equivariant cobordant. Compos. Math. 56:2 (1985), 171-200.

[Go12] Gordon C. Orbifolds and their spectra. In Spectral Geometry, 49-71, Proc. Sympos. Pure Math. 84, Amer. Math. Soc., Providence, RI, 2012. DOI: 10.1090/pspum/084.

[GR03] GoRdon C.S., Rossetti J.P. Boundary volume and length spectra of Riemannian manifolds: what the middle degree Hodge spectrum doesn't reveal. Ann. Inst. Fourier (Grenoble) 53:7 (2003), 2297-2314. DOI: 10.5802/aif.2007. 
[GM06] Gornet R., McGowan J. Lens Spaces, isospectral on forms but not on functions. LMS J. Comput. Math. 9 (2006), 270-286. DOI: 10.1112/S1461157000001273.

[Ha11] Hattori T. On the residues of spectral zeta functions on spheres. Saitama Math. J. 28 (2011), 25-37.

[He1] Helgason S. Groups and geometric analysis. Integral geometry, invariant differential operators, and spherical functions. Math. Surveys Monogr 83. Amer. Math. Soc., Providence, 2000.

[He2] Helgason S. Differential geometry, Lie groups, and symmetric spaces. 34. Amer. Math. Soc., Providence, 2001.

[Ik80a] IKEDA A. On lens spaces which are isospectral but not isometric. Ann. Sci. École Norm. Sup. (4) 13:3 (1980), 303-315.

[Ik80b] IKEDA A. On the spectrum of a riemannian manifold of positive constant curvature. Osaka J. Math. 17 (1980), 75-93.

[Ik80c] IKEDA A. On the spectrum of a riemannian manifold of positive constant curvature II. Osaka J. Math. 17 (1980), 691-762.

[Ik83] IKEDA A. On spherical space forms which are isospectral but not isometric. J. Math. Soc. Japan 35:3 (1983), 437-444. DOI: 10.2969/jmsj/03530437.

[Ik88] IKEDA A. Riemannian manifolds p-isospectral but not $p+1$-isospectral. In Geometry of manifolds (Matsumoto, 1988), 383-417, Perspect. Math. 8, 1989.

[Ik97] IKEDA A. On space forms of real Grassmann manifolds which are isospectral but not isometric. Kodai Math. J. 20:1 (1997), 1-7. DOI: $10.2996 / \mathrm{kmj} / 1138043715$

[IT78] Ikeda A., TAniguchi Y. Spectra and eigenforms of the Laplacian on $S^{n}$ and $P^{n}(\mathbb{C})$. Osaka J. Math. 15:3 (1978), 515-546.

[IY79] IkedA A., Yамамото Y. On the spectra of 3-dimensional lens spaces. Osaka J. Math. 16:2 (1979), 447-469.

[Kn] KNAPP A. W. Lie groups beyond an introduction. Progress in Mathematics 140. Birkhäuser Boston Inc., 2002.

[LMR13] Lauret E.A., Miatello R.J., Rossetti, J.P. Strongly isospectral manifolds with nonisomorphic cohomology rings. Rev. Mat. Iberoam. 29 (2013), 611-634. DOI: 10.4171/RMI/732.

[LMR15] Lauret E.A., Miatello R.J., Rossetti J.P. Spectra of lens spaces from 1-norm spectra of congruence lattices. In press, Int. Math. Res. Not. IMRN, published electronically in June 2015. DOI: $10.1093 / \mathrm{imrn} / \mathrm{rnv} 159$

[LMR16] Lauret E.A., Miatello R.J., Rossetti J.P. Non-strongly isospectral spherical space forms. In Mathematical Congress of the Americas, Contemp. Math. 656, Amer. Math. Soc., Providence, RI, 2016. DOI: $10.1090 / \mathrm{conm} / 656 / 13104$

[MR02] Miatello R.J., Rossetti J.P. Comparison of twisted Laplace p-spectra for flat manifolds with diagonal holonomy. Ann. Global Anal. Geom. 21:4 (2002), 341-376. DOI: 10.1023/A:1015651821995

[Mi64] Milnor J. Eigenvalues of the Laplace operator on certain manifolds. Proc. Natl. Acad. Sci. USA $\mathbf{5 1 : 4}(1964), 542$.

[MH16] MOHADES H, HonARI B. On a relation between spectral theory of lens spaces and Ehrhart theory. arXiv:1601.04256 (2016).

[Pe96] PEscE H. Représentations relativement équivalentes et variétés riemanniennes isospectrales. Comment. Math. Helvetici 71 (1996), 243-268. DOI: 10.1007/BF02566419.

[RSW08] Rossetti J.P., Schueth D., Weilandt M. Isospectral orbifolds with different maximal isotropy orders. Ann. Global Anal. Geom. 34:4 (2008), 351-366. DOI: 10.1007/s10455-008-9110-3.

[Sage] Stein W. A. ET AL. Sage Mathematics Software (Version 4.3). The Sage Development Team, 2009, www.sagemath.org.

[Sh11] Shams Ul BARI N. Orbifold lens spaces that are isospectral but not isometric. Osaka J. Math, 48:1 (2011), 1-40.

[SSW06] Shams N., Stanhope E., WebB D. One cannot hear orbifold isotropy type. Arch. Math. (Basel) 87:4 (2006), 375-385. DOI: 10.1007/s00013-006-1748-0.

[Sp03] SPREAFICO M. Zeta functions and regularized determinants on projective spaces. Rocky Mountain J. Math. 33:4 (2003), 1499-1512. DOI: 10.1216/rmjm/1181075478.

[Teo14] TEO L.P. Zeta functions of spheres and real projective spaces. arXiv:1412.0758 (2014).

[Wa73] Wallach N.R. Harmonic analysis on homogeneous spaces. Pure and Applied Mathematics 19. Marcel Dekker, Inc., New York, 1973.

[Wo01] Wolf J.A. Isospectrality for spherical space forms. Result. Math. 40 (2001), 321-338. DOI: $10.1007 / \mathrm{BF} 03322715$. 
[Ya80] Yамамото Y. On the number of lattice points in the square $x+y \leq u$ with a certain congruence condition. Osaka J. Math. 17:1 (1980), 9-21.

CiEm-FamaF, Universidad Nacional de Córdoba, 5000-Córdoba, Argentina.

E-mail address: elauret@famaf.unc.edu.ar 